\title{
T-Systems and Y-Systems for Quantum Affinizations of Quantum Kac-Moody Algebras
}

\author{
Atsuo KUNIBA ${ }^{\dagger}$, Tomoki NAKANISHI ${ }^{\ddagger}$ and Junji SUZUKI ${ }^{\S}$ \\ $\dagger$ Institute of Physics, University of Tokyo, Tokyo, 153-8902, Japan \\ E-mail: atsuo@gokutan.c.u-tokyo.ac.jp \\ $\ddagger$ Graduate School of Mathematics, Nagoya University, Nagoya, 464-8604, Japan \\ E-mail: nakanisi@math.nagoya-u.ac.jp \\ $\S$ Department of Physics, Faculty of Science, Shizuoka University, Ohya, 836, Japan \\ E-mail: sjsuzuk@ipc.shizuoka.ac.jp
}

Received October 05, 2009, in final form December 16, 2009; Published online December 19, 2009 doi:10.3842/SIGMA.2009.108

\begin{abstract}
The T-systems and Y-systems are classes of algebraic relations originally associated with quantum affine algebras and Yangians. Recently the T-systems were generalized to quantum affinizations of a wide class of quantum Kac-Moody algebras by Hernandez. In this note we introduce the corresponding $\mathrm{Y}$-systems and establish a relation between $\mathrm{T}$ and Y-systems. We also introduce the $\mathrm{T}$ and $\mathrm{Y}$-systems associated with a class of cluster algebras, which include the former $\mathrm{T}$ and $\mathrm{Y}$-systems of simply laced type as special cases.
\end{abstract}

Key words: T-systems; Y-systems; quantum groups; cluster algebras

2000 Mathematics Subject Classification: 17B37; 13A99

Dedicated to Professor Tetsuji Miwa on his 60th birthday

\section{Introduction}

The T-systems and Y-systems appear in various aspects for integrable systems [58, 39, 40, 42, $52,43,44,22,2,41,6,13,1,12,53,24,55]$. Originally, the T-systems are systems of relations among the Kirillov-Reshetikhin modules [37, 38] in the Grothendieck rings of modules over quantum affine algebras and Yangians [3, 7, 43, 45, 8, 20, 48, 25, 28]. The T and Y-systems are related to each other by certain changes of variables [40, 43].

The $\mathrm{T}$ and $\mathrm{Y}$-systems are also regarded as relations among variables for cluster algebras $[17,19,29,10,36,32,30,49,11]$. This identification is especially fruitful in the study of the periodicity of these systems [58, 52, 43, 22, 21, 17, 18, 19, 57, 36, 32].

The T-systems are generalized by Hernandez [27] to the quantum affinizations of a wide class of quantum Kac-Moody algebras studied in [15, 56, 34, 46, 47, 26]. In this paper we introduce the corresponding Y-systems and establish a relation between $\mathrm{T}$ and $\mathrm{Y}$-systems. We also introduce the $\mathrm{T}$ and $\mathrm{Y}$-systems associated with a class of cluster algebras, which include the former $\mathrm{T}$ and $\mathrm{Y}$-systems of simply laced type as special cases.

It will be interesting to investigate the relation of the systems discussed here to the birational transformations arising from the Painlevé equations in $[50,51]$, and also to the geometric realization of cluster algebras in [5, 23].

The organization of the paper is as follows. In Section 2 basic definitions for quantum Kac-Moody algebras $U_{q}(\mathfrak{g})$ and their quantum affinizations $U_{q}(\hat{\mathfrak{g}})$ are recalled. In Section 3 the T-systems associated with the quantum affinizations of a class of quantum Kac-Moody algebras by [27] are presented. Based on the result by [27], the role of the T-system in the 
A. Kuniba, T. Nakanishi and J. Suzuki

Grothendieck ring of $U_{q}(\hat{\mathfrak{g}})$-modules is given (Corollary 3.8). In Section 4 we introduce the Y-systems corresponding to the T-systems in Section 3, and establish a relation between them (Theorem 4.4). In Section 5 we define the restricted version of T-systems and Y-systems, and establish a relation between them (Theorem 5.3). In Section 6 we introduce the T and Y-systems associated with a class of cluster algebras, which include the restricted $\mathrm{T}$ and $\mathrm{Y}$-systems of simply laced type as special cases. In particular, the correspondence between the restricted $\mathrm{T}$ and $\mathrm{Y}$ systems of simply laced type for the quantum affinizations and cluster algebras is presented (Corollaries 6.20, 6.21, 6.25, and 6.26).

\section{Quantum Kac-Moody algebras and their quantum affinizations}

In this section, we recall basic definitions for quantum Kac-Moody algebras and their quantum affinizations, following $[26,27]$. The presentation here is a minimal one. See [26, 27] for further information and details.

\subsection{Quantum Kac-Moody algebras}

Let $I=\{1, \ldots, r\}$ and let $C=\left(C_{i j}\right)_{i, j \in I}$ be a generalized Cartan matrix in [35]; namely, it satisfies $C_{i j} \in \mathbb{Z}, C_{i i}=2, C_{i j} \leq 0$ for any $i \neq j$, and $C_{i j}=0$ if and only if $C_{j i}=0$. We assume that $C$ is symmetrizable, i.e., there is a diagonal matrix $D=\operatorname{diag}\left(d_{1}, \ldots, d_{r}\right)$ with $d_{i} \in \mathbb{N}:=\mathbb{Z}_{>0}$ such that $B=D C$ is symmetric. Throughout the paper we assume that there is no common divisor for $d_{1}, \ldots, d_{r}$ except for 1 .

Let $\left(\mathfrak{h}, \Pi, \Pi^{\vee}\right)$ be a realization of the Cartan matrix $C[35]$; namely, $\mathfrak{h}$ is a $(2 r-\operatorname{rank} C)$ dimensional $\mathbb{Q}$-vector space, and $\Pi=\left\{\alpha_{1}, \ldots, \alpha_{r}\right\} \subset \mathfrak{h}^{*}, \Pi^{\vee}=\left\{\alpha_{1}^{\vee}, \ldots, \alpha_{r}^{\vee}\right\} \subset \mathfrak{h}$ such that $\alpha_{j}\left(\alpha_{i}^{\vee}\right)=C_{i j}$.

Let $q \in \mathbb{C}^{\times}$be not a root of unity. We set $q_{i}=q^{d_{i}}(i \in I),[k]_{q}=\left(q^{k}-q^{-k}\right) /\left(q-q^{-1}\right)$, $[k]_{q} !=[1]_{q}[2]_{q} \cdots[k]_{q}$, and $\left[\begin{array}{l}k \\ r\end{array}\right]_{q}=[k]_{q} ! /[k-r]_{q} ![r]_{q} !(0 \leq r \leq k)$.

Definition $2.1([14,33])$. The quantum Kac-Moody algebra $U_{q}(\mathfrak{g})$ associated with $C$ is the $\mathbb{C}$-algebra with generators $k_{h}(h \in \mathfrak{h}), x_{i}^{ \pm}(i \in I)$ and the following relations:

$$
\begin{aligned}
& k_{h} k_{h^{\prime}}=k_{h+h^{\prime}}, \quad k_{0}=1, \quad k_{h} x_{i}^{ \pm} k_{-h}=q^{ \pm \alpha_{i}(h)} x_{i}^{ \pm}, \\
& x_{i}^{+} x_{j}^{-}-x_{j}^{-} x_{i}^{+}=\delta_{i j} \frac{k_{d_{i} \alpha_{i}^{\vee}}-k_{-d_{i} \alpha_{i}^{\vee}}}{q_{i}-q_{i}^{-1}}, \\
& \sum_{r=0}^{1-C_{i j}}(-1)^{r}\left[\begin{array}{c}
1-C_{i j} \\
r
\end{array}\right]_{q_{i}}\left(x_{i}^{ \pm}\right)^{1-C_{i j}-r} x_{j}^{ \pm}\left(x_{i}^{ \pm}\right)^{r}=0 \quad(i \neq j) .
\end{aligned}
$$

\subsection{Quantum affinizations}

In the following, we use the following formal series (currents):

$$
\begin{aligned}
& x_{i}^{ \pm}(z)=\sum_{r \in \mathbb{Z}} x_{i, r}^{ \pm} z^{r}, \\
& \phi_{i}^{ \pm}(z)=\sum_{r \geq 0} \phi_{i, \pm r}^{ \pm} z^{ \pm r}:=k_{ \pm d_{i} \alpha_{i}^{\vee}} \exp \left( \pm\left(q-q^{-1}\right) \sum_{r \geq 1} h_{i, \pm r} z^{ \pm r}\right) .
\end{aligned}
$$

We also use the formal delta function $\delta(z)=\sum_{r \in \mathbb{Z}} z^{r}$. 
Definition 2.2 ([15, 34, 27]). The quantum affinization (without central elements) of the quantum Kac-Moody algebra $U_{q}(\mathfrak{g})$, denoted by $U_{q}(\hat{\mathfrak{g}})$, is the $\mathbb{C}$-algebra with generators $x_{i, r}^{ \pm}$ $(i \in I, r \in \mathbb{Z}), k_{h}(h \in \mathfrak{h}), h_{i, r}(i \in I, r \in \mathbb{Z} \backslash\{0\})$ and the following relations:

$$
\begin{aligned}
& k_{h} k_{h^{\prime}}=k_{h+h^{\prime}}, \quad k_{0}=1, \quad k_{h} \phi_{i}^{ \pm}(z)=\phi_{i}^{ \pm}(z) k_{h}, \\
& k_{h} x_{i}^{ \pm}(z)=q^{ \pm \alpha_{i}(h)} x_{i}^{ \pm}(z) k_{h}, \\
& \phi_{i}^{+}(z) x_{j}^{ \pm}(w)=\frac{q^{ \pm B_{i j}} w-z}{w-q^{ \pm B_{i j} z}} x_{j}^{ \pm}(w) \phi_{i}^{+}(z), \\
& \phi_{i}^{-}(z) x_{j}^{ \pm}(w)=\frac{q^{ \pm B_{i j}} w-z}{w-q^{ \pm B_{i j}} z} x_{j}^{ \pm}(w) \phi_{i}^{-}(z), \\
& x_{i}^{+}(z) x_{j}^{-}(w)-x_{j}^{-}(w) x_{i}^{+}(z)=\frac{\delta_{i j}}{q_{i}-q_{i}^{-1}}\left(\delta\left(\frac{w}{z}\right) \phi_{i}^{+}(w)-\delta\left(\frac{z}{w}\right) \phi_{i}^{-}(z)\right), \\
& \left(w-q^{ \pm B_{i j}} z\right) x_{i}^{ \pm}(z) x_{j}^{ \pm}(w)=\left(q^{ \pm B_{i j}} w-z\right) x_{j}^{ \pm}(w) x_{i}^{ \pm}(z), \\
& \sum_{\pi \in \Sigma} \sum_{k=1}^{1-C_{i j}}(-1)^{k}\left[\begin{array}{c}
1-C_{i j} \\
k
\end{array}\right]_{q_{i}} x_{i}^{ \pm}\left(w_{\pi(1)}\right) \cdots x_{i}^{ \pm}\left(w_{\pi(k)}\right) x_{j}^{ \pm}(z) \\
& \quad \times x_{i}^{ \pm}\left(w_{\pi(k+1)}\right) \cdots x_{i}^{ \pm}\left(w_{\pi\left(1-C_{i j}\right)}\right)=0 \quad(i \neq j) .
\end{aligned}
$$

In (2.1) $\Sigma$ is the symmetric group for the set $\left\{1, \ldots, 1-C_{i j}\right\}$.

When $C$ is of finite type, the above $U_{q}(\hat{\mathfrak{g}})$ is called an (untwisted) quantum affine algebra (without central elements) or quantum loop algebra; it is isomorphic to a subquotient of the quantum Kac-Moody algebra associated with the (untwisted) affine extension of $C$ without derivation and central elements [15, 4]. (A little confusingly, the quantum Kac-Moody algebra associated with $C$ of affine type with derivation and central elements is also called a quantum affine algebra and denoted by $U_{q}(\hat{\mathfrak{g}})$.)

When $C$ is of affine type, $U_{q}(\hat{\mathfrak{g}})$ is called a quantum toroidal algebra (without central elements).

In general, if $C$ is not of finite type, $U_{q}(\hat{\mathfrak{g}})$ is no longer isomorphic to a subquotient of any quantum Kac-Moody algebra and has no Hopf algebra structure.

\subsection{The category $\operatorname{Mod}\left(U_{q}(\hat{\mathfrak{g}})\right)$}

Let $U_{q}(\mathfrak{h})$ be the subalgebra of $U_{q}(\hat{\mathfrak{g}})$ generated by $k_{h}(h \in \mathfrak{h})$.

Definition $2.3([27])$. Let $\operatorname{Mod}\left(U_{q}(\hat{\mathfrak{g}})\right)$ be the category of $U_{q}(\hat{\mathfrak{g}})$-modules satisfying the following properties:

(a) $V$ is $U_{q}(\mathfrak{h})$-diagonalizable, i.e., $V=\oplus_{\omega \in \mathfrak{h}^{*}} V_{\omega}$, where

$$
V_{\omega}=\left\{v \in V \mid k_{h} v=q^{\omega(h)} v \text { for any } h \in \mathfrak{h}\right\} .
$$

(b) For any $\omega \in \mathfrak{h}^{*}, V_{\omega}$ is finite dimensional.

(c) For any $i \in I$ and $\omega \in \mathfrak{h}^{*}, V_{\omega \pm r \alpha_{i}}=\{0\}$ for a sufficiently large $r$.

(d) There is a finite number of elements $\lambda_{1}, \ldots, \lambda_{s} \in \mathfrak{h}^{*}$ such that the weights $\omega \in \mathfrak{h}^{*}$ of $V$ are in $\bigcup_{j=1}^{s} \mathcal{S}\left(\lambda_{j}\right)$, where $\mathcal{S}(\lambda)=\left\{\mu \in \mathfrak{h}^{*} \mid \mu \leq \lambda\right\}$.

By Condition $(a)$, we restrict our attention to the so called 'type 1' modules.

Let

$$
P=\left\{\lambda \in \mathfrak{h}^{*} \mid \lambda\left(\alpha_{i}^{\vee}\right) \in \mathbb{Z}\right\}
$$

be the set of integral weights. 
Definition 2.4 ([26]).

(1) An $\ell$-weight is a pair $(\lambda, \Psi)$ with $\lambda \in P$ and $\Psi=\left(\Psi_{i, \pm r}^{ \pm}\right)_{i \in I, r \geq 0}$ such that $\Psi_{i, \pm r}^{ \pm} \in \mathbb{C}$ and $\Psi_{i, 0}^{ \pm}=q_{i}^{ \pm \lambda\left(\alpha_{i}^{\vee}\right)}$

(2) A $U_{q}(\hat{\mathfrak{g}})$-module $V$ is of $\ell$-highest weight if there is some $v \in V$ and $\ell$-weight $(\lambda, \Psi)$ such that $x_{i, r}^{+} v=0, k_{h} v=q^{\lambda(h)} v, \phi_{i, \pm r}^{ \pm} v=\Psi_{i, \pm r}^{ \pm} v$, and $U_{q}(\hat{\mathfrak{g}}) v=V$. Such $v$ and $(\lambda, \Psi)$ are called a highest weight vector and the $\ell$-highest weight of $V$, respectively.

By the standard argument using Verma modules, one can show that for any $\ell$-weight $(\lambda, \Psi)$, there is a unique simple $\ell$-highest weight module $L(\lambda, \Psi)$ with $\ell$-highest weight $(\lambda, \Psi)[26]$.

The following theorem is a generalization of the well-known classification of the simple finitedimensional modules of the quantum affine algebras by $[7,8]$.

Theorem $2.5([46,47,26])$. We have $L(\lambda, \Psi) \in \operatorname{Mod}\left(U_{q}(\hat{\mathfrak{g}})\right)$ if and only if there is an I-tuple of polynomials $\left(P_{i}(u)\right)_{i \in I}, P_{i}(u) \in \mathbb{C}[u]$ with $P_{i}(0)=1$ such that

$$
\sum_{m \geq 0} \Psi_{i, \pm m}^{ \pm} z^{ \pm m}=q_{i}^{\operatorname{deg} P_{i}} \frac{P_{i}\left(z q_{i}^{-1}\right)}{P_{i}\left(z q_{i}\right)}
$$

where the equality is in $\mathbb{C}\left[\left[z^{ \pm 1}\right]\right]$.

We call $\left(P_{i}(u)\right)_{i \in I}$ the Drinfeld polynomials of $L(\lambda, \Psi)$. In the case of quantum affine algebras, $\lambda$ is also completely determined by the Drinfeld polynomials by the condition $\lambda\left(\alpha_{i}^{\vee}\right)=\operatorname{deg} P_{i}$. This is not so in general.

Let $\Lambda_{i} \in \mathfrak{h}^{*}(i \in I)$ be the fundamental weights of $U_{q}(\mathfrak{g})$ satisfying $\Lambda_{i}\left(\alpha_{j}^{\vee}\right)=\delta_{i j}$.

Example 2.6. For the following choices of $(\lambda, \Psi), L(\lambda, \Psi) \in \operatorname{Mod}\left(U_{q}(\hat{\mathfrak{g}})\right)$ is called a fundamental module [27].

(a) For any $i \in I$ and $\alpha \in \mathbb{C}^{\times}$, set $\lambda=\Lambda_{i}, P_{i}(u)=1-\alpha u$, and $P_{j}(u)=1(j \in I, j \neq i)$.

(b) Choose any $\lambda$ satisfying $\left(\lambda, \alpha_{i}^{\vee}\right)=0(i \in I)$ and also set $P_{i}(u)=1(i \in I)$. The corresponding module $L(\lambda, \Psi)$ is written as $L(\lambda)$. The module $L(\lambda)$ is one-dimensional; it is trivial in the case of the quantum affine algebras.

\subsection{Kirillov-Reshetikhin modules}

The following is a generalization of the Kirillov-Reshetikhin modules of the quantum affine algebras studied by [37, 38, 3, 8, 43, 9].

Definition $2.7([27])$. For any $i \in I, m \in \mathbb{N}$, and $\alpha \in \mathbb{C}^{\times}$, set the polynomials $\left(P_{j}(u)\right)_{j \in I}$ as

$$
P_{i}(u)=\left(1-\alpha q_{i}^{m-1} u\right)\left(1-\alpha q_{i}^{m-3} u\right) \cdots\left(1-\alpha q_{i}^{1-m} u\right)
$$

and $P_{j}(u)=1$ for any $j \neq i$. The corresponding module $L\left(m \Lambda_{i}, \Psi\right)$ is called a KirillovReshetikhin module and denoted by $W_{m, \alpha}^{(i)}$.

Remark 2.8. We slightly shift the definition of the polynomial (2.2) for $W_{m, \alpha}^{(i)}$ in [27] in order to make the identification to the forthcoming T-systems a little simpler.

\section{T-systems}

\section{$3.1 \quad$ T-systems}

Throughout Sections 3-5, we restrict our attention to a symmetrizable generalized Cartan matrix $C$ satisfying the following condition due to Hernandez [27]:

$$
\text { If } C_{i j}<-1 \text {, then } d_{i}=-C_{j i}=1 \text {, }
$$


where $D=\operatorname{diag}\left(d_{1}, \ldots, d_{r}\right)$ is the diagonal matrix symmetrizing $C$. In this paper, we say that a generalized Cartan matrix $C$ is tamely laced if it is symmetrizable and satisfies the condition (3.1).

As usual, we say that a generalized Cartan matrix $C$ is simply laced if $C_{i j}=0$ or -1 for any $i \neq j$. If $C$ is simply laced, then it is symmetric, $d_{a}=1$ for any $a \in I$, and it is tamely laced.

With a tamely laced generalized Cartan matrix $C$, we associate a Dynkin diagram in the standard way [35]: For any pair $i \neq j \in I$ with $C_{i j}<0$, the vertices $i$ and $j$ are connected by $\max \left\{\left|C_{i j}\right|,\left|C_{j i}\right|\right\}$ lines, and the lines are equipped with an arrow from $j$ to $i$ if $C_{i j}<-1$. Note that the condition (3.1) means

(i) the vertices $i$ and $j$ are not connected if $d_{i}, d_{j}>1$ and $d_{i} \neq d_{j}$,

(ii) the vertices $i$ and $j$ are connected by $d_{i}$ lines with an arrow from $i$ to $j$ or not connected if $d_{i}>1$ and $d_{j}=1$,

(iii) the vertices $i$ and $j$ are connected by a single line or not connected if $d_{i}=d_{j}$.

Example 3.1. (1) Any Cartan matrix of finite or affine type is tamely laced except for types $A_{1}^{(1)}$ and $A_{2 \ell}^{(2)}$.

(2) The following generalized Cartan matrix $C$ is tamely laced:

$$
C=\left(\begin{array}{cccc}
2 & -1 & 0 & 0 \\
-3 & 2 & -2 & -2 \\
0 & -1 & 2 & -1 \\
0 & -1 & -1 & 2
\end{array}\right), \quad D=\left(\begin{array}{cccc}
3 & 0 & 0 & 0 \\
0 & 1 & 0 & 0 \\
0 & 0 & 2 & 0 \\
0 & 0 & 0 & 2
\end{array}\right)
$$

The corresponding Dynkin diagram is

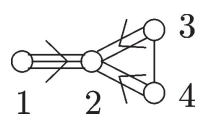

For a tamely laced generalized Cartan matrix $C$, we set an integer $t$ by

$$
t=\operatorname{lcm}\left(d_{1}, \ldots, d_{r}\right) .
$$

For $a, b \in I$, we write $a \sim b$ if $C_{a b}<0$, i.e., $a$ and $b$ are adjacent in the corresponding Dynkin diagram.

Let $U$ be either $\frac{1}{t} \mathbb{Z}$, the complex plane $\mathbb{C}$, or the cylinder $\mathbb{C}_{\xi}:=\mathbb{C} /(2 \pi \sqrt{-1} / \xi) \mathbb{Z}$ for some $\xi \in$ $\mathbb{C} \backslash 2 \pi \sqrt{-1} \mathbb{Q}$, depending on the situation under consideration. The following is a generalization of the T-systems associated with the quantum affine algebras [43].

Definition 3.2 ([27]). For a tamely laced generalized Cartan matrix $C$, the unrestricted $T$ system $\mathbb{T}(C)$ associated with $C$ is the following system of relations for a family of variables $T=\left\{T_{m}^{(a)}(u) \mid a \in I, m \in \mathbb{N}, u \in U\right\}$,

$$
\begin{aligned}
& T_{m}^{(a)}\left(u-\frac{d_{a}}{t}\right) T_{m}^{(a)}\left(u+\frac{d_{a}}{t}\right)=T_{m-1}^{(a)}(u) T_{m+1}^{(a)}(u)+\prod_{b: b \sim a} T_{\frac{d_{a}}{d_{b}}}^{(b)}(u) \quad \text { if } \quad d_{a}>1, \\
& T_{m}^{(a)}\left(u-\frac{d_{a}}{t}\right) T_{m}^{(a)}\left(u+\frac{d_{a}}{t}\right)=T_{m-1}^{(a)}(u) T_{m+1}^{(a)}(u)+\prod_{b: b \sim a} S_{m}^{(b)}(u) \quad \text { if } \quad d_{a}=1,
\end{aligned}
$$

where $T_{0}^{(a)}(u)=1$ if they occur in the right hand sides in the relations. The symbol $S_{m}^{(b)}(u)$ is defined by

$$
S_{m}^{(b)}(u)=\prod_{k=1}^{d_{b}} T_{1+E\left[\frac{m-k}{d_{b}}\right]}^{(b)}\left(u+\frac{1}{t}\left(2 k-1-m+E\left[\frac{m-k}{d_{b}}\right] d_{b}\right)\right),
$$

and $E[x](x \in \mathbb{Q})$ denotes the largest integer not exceeding $x$. 


\section{Remark 3.3.}

1. This is a slightly reduced version of the T-systems in [27, Theorem 6.10]. See Remark 3.7. The same system was also studied by [54] when $C$ is of affine type in view of a generalization of discrete Toda field equations.

2. More explicitly, $S_{m}^{(b)}(u)$ is written as follows: For $0 \leq j<d_{b}$,

$$
S_{d_{b} m+j}^{(b)}(u)=\left\{\prod_{k=1}^{j} T_{m+1}^{(b)}\left(u+\frac{1}{t}(j+1-2 k)\right)\right\}\left\{\prod_{k=1}^{d_{b}-j} T_{m}^{(b)}\left(u+\frac{1}{t}\left(d_{b}-j+1-2 k\right)\right)\right\} .
$$

For example, for $d_{b}=1$,

$$
S_{m}^{(b)}(u)=T_{m}^{(b)}(u)
$$

for $d_{b}=2$,

$$
\begin{aligned}
& S_{2 m}^{(b)}(u)=T_{m}^{(b)}\left(u-\frac{1}{t}\right) T_{m}^{(b)}\left(u+\frac{1}{t}\right), \\
& S_{2 m+1}^{(b)}(u)=T_{m+1}^{(b)}(u) T_{m}^{(b)}(u),
\end{aligned}
$$

for $d_{b}=3$,

$$
\begin{aligned}
& S_{3 m}^{(b)}(u)=T_{m}^{(b)}\left(u-\frac{2}{t}\right) T_{m}^{(b)}(u) T_{m}^{(b)}\left(u+\frac{2}{t}\right), \\
& S_{3 m+1}^{(b)}(u)=T_{m+1}^{(b)}(u) T_{m}^{(b)}\left(u-\frac{1}{t}\right) T_{m}^{(b)}\left(u+\frac{1}{t}\right), \\
& S_{3 m+2}^{(b)}(u)=T_{m+1}^{(b)}\left(u-\frac{1}{t}\right) T_{m+1}^{(b)}\left(u+\frac{1}{t}\right) T_{m}^{(b)}(u),
\end{aligned}
$$

and so on.

3. The second terms in the right hand sides of (3.2) and (3.3) can be written in a unified way as follows [27]:

$$
\prod_{b: b \sim a} \prod_{k=1}^{-C_{a b}} T_{-C_{b a}+E\left[\frac{d_{a}(m-k)}{d_{b}}\right]}^{(b)}\left(u+\frac{d_{b}}{t}\left(\frac{-2 k+1}{C_{a b}}-C_{b a}+E\left[\frac{d_{a}(m-k)}{d_{b}}\right]-1\right)-\frac{d_{a} m}{t}\right) .
$$

Definition 3.4. Let $\mathcal{T}(C)$ be the commutative ring over $\mathbb{Z}$ with generators $T_{m}^{(a)}(u)^{ \pm 1}(a \in I$, $m \in \mathbb{N}, u \in U)$ and the relations $\mathbb{T}(C)$. (Here we also assume the relation $T_{m}^{(a)}(u) T_{m}^{(a)}(u)^{-1}=1$ implicitly. We do not repeat this remark in the forthcoming similar definitions.) Also, let $\mathcal{T}^{\circ}(C)$ be the subring of $\mathcal{T}(C)$ generated by $T_{m}^{(a)}(u)(a \in I, m \in \mathbb{N}, u \in U)$.

\subsection{T-system and Grothendieck ring}

Let $C$ continue to be a tamely laced generalized Cartan matrix. The T-system $\mathbb{T}(C)$ is a family of relations in the Grothendieck ring of modules of $U_{q}(\hat{\mathfrak{g}})$ as explained below.

We recall facts on $\operatorname{Mod}\left(U_{q}(\hat{\mathfrak{g}})\right)$ in [27].

1. For a pair of $\ell$-highest weight modules $V_{1}, V_{2} \in \operatorname{Mod}\left(U_{q}(\hat{\mathfrak{g}})\right)$, there is an $\ell$-highest weight module $V_{1} *_{f} V_{2} \in \operatorname{Mod}\left(U_{q}(\hat{\mathfrak{g}})\right)$ called the fusion product. It is defined by using the $u$ deformation of the Drinfeld coproduct and the specialization at $u=1$.

2. Any $\ell$-highest weight module in $\operatorname{Mod}\left(U_{q}(\hat{\mathfrak{g}})\right)$ has a finite composition. (Here, the condition (3.1) for $C$ is used essentially in [27].) 
3. If $V_{1}, V_{2} \in \operatorname{Mod}\left(U_{q}(\hat{\mathfrak{g}})\right)$ have finite compositions, then $V_{1} *_{f} V_{2}$ also has a finite composition.

Therefore, the Grothendieck ring $R(C)$ of the modules in $\operatorname{Mod}\left(U_{q}(\hat{\mathfrak{g}})\right)$ having finite compositions is well defined, where the product is given by $*_{f}$.

Let $R^{\prime}(C)$ be the quotient ring of $R(C)$ by the ideal generated by all $L(\lambda, \Psi)-L\left(\lambda^{\prime}, \Psi\right)^{\prime}$ 's. In other words, we regard modules in $R(C)$ as modules of the subalgebra of $U_{q}(\hat{\mathfrak{g}})$ generated by $x_{i, r}^{ \pm}$ $(i \in I, r \in \mathbb{Z}), k_{ \pm d_{i} \alpha_{i}^{\vee}}(i \in I), h_{i, r}(i \in I, r \in \mathbb{Z} \backslash\{0\})$ in Definition 2.2.

Proposition 3.5. The ring $R^{\prime}(C)$ is freely generated by the fundamental modules $L\left(\Lambda_{i}, \Psi\right)$ in Example $2.6(a)$.

Proof. It follows from [27, Corollary 4.9] that $R^{\prime}(C)$ is generated by the fundamental modules $L\left(\Lambda_{i}, \Psi\right)$. The $q$-character morphism $\chi_{q}$ defined in [27] induces an injective ring homomorphism

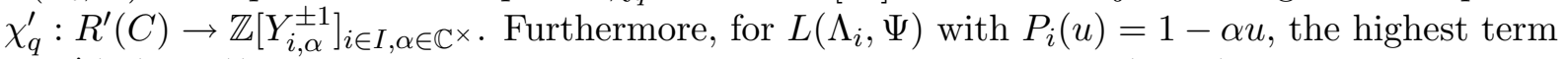
of $\chi_{q}^{\prime}\left(L\left(\Lambda_{i}, \Psi\right)\right)$ is $Y_{i, \alpha}$. Therefore, the algebraic independence of $L\left(\Lambda_{i}, \Psi\right)$ 's follows from that of $Y_{i, \alpha}$ 's.

We set $\mathbb{C}_{t \log q}:=\mathbb{C} /(2 \pi \sqrt{-1} /(t \log q)) \mathbb{Z}$, and introduce alternative notation $W_{m}^{(a)}(u)(a \in I$, $m \in \mathbb{N}, u \in \mathbb{C}_{t \log q}$ ) for the Kirillov-Reshetikhin module $W_{m, q^{t u}}^{(a)}$ in Definition 2.7.

In terms of the Kirillov-Reshetikhin modules, the structure of $R^{\prime}(C)$ is described as follows:

Theorem 3.6. Let $W=\left\{W_{m}^{(a)}(u) \mid a \in I, m \in \mathbb{N}, u \in \mathbb{C}_{t \log q}\right\}$ be the family of the KirillovReshetikhin modules in $R^{\prime}(C)$. Let $T$ and $\mathbb{T}(C)$ be the ones in Definition 3.4 with $U=\mathbb{C}_{t \log q}$. Then,

(1) The family $W$ generates the ring $R^{\prime}(C)$.

(2) ([27]) The family $W$ satisfies the T-system $\mathbb{T}(C)$ in $R^{\prime}(C)$ by replacing $T_{m}^{(a)}(u)$ in $\mathbb{T}(C)$ with $W_{m}^{(a)}(u)$.

(3) For any polynomial $P(T) \in \mathbb{Z}[T]$, the relation $P(W)=0$ holds in $R^{\prime}(C)$ if and only if there is a nonzero monomial $M(T) \in \mathbb{Z}[T]$ such that $M(T) P(T) \in I(\mathbb{T}(C)$ ), where $I(\mathbb{T}(C))$ is the ideal of $\mathbb{Z}[T]$ generated by the relations in $\mathbb{T}(C)$.

Proof. (1) By Proposition 3.5, $R^{\prime}(C)$ is generated by $L\left(\Lambda_{i}, \Psi\right)$ 's, which belong to $W$.

(2) This is due to [27, Theorem 6.10].

(3) The proof is the same with that of [32, Theorem 2.8] by generalizing the height of $T_{m}^{(a)}(u)$ therein as $\operatorname{ht} T_{m}^{(a)}(u):=d_{a}(m-1)+1$.

Remark 3.7. In [27] the T-system is considered in $R(C)$ including fundamental modules $L(\lambda)$ of Example $2.6(b)$.

As a corollary, we have a generalization of [32, Corollary 2.9] for the quantum affine algebras:

Corollary 3.8. The ring $\mathcal{T}^{\circ}(C)$ with $U=\mathbb{C}_{t \log q}$ is isomorphic to $R^{\prime}(C)$ by the correspondence $T_{m}^{(a)}(u) \mapsto W_{m}^{(a)}(u)$.

\section{$4 \quad$ Y-systems}

\subsection{Y-systems}

Definition 4.1. For a tamely laced generalized Cartan matrix $C$, the unrestricted $Y$-system $\mathbb{Y}(C)$ associated with $C$ is the following system of relations for a family of variables $Y=\left\{Y_{m}^{(a)}(u) \mid\right.$ 
$a \in I, m \in \mathbb{N}, u \in U\}$, where $Y_{0}^{(a)}(u)^{-1}=0$ if they occur in the right hand sides in the relations:

$$
\begin{aligned}
& Y_{m}^{(a)}\left(u-\frac{d_{a}}{t}\right) Y_{m}^{(a)}\left(u+\frac{d_{a}}{t}\right)=\frac{\prod_{b: b \sim a} Z_{\frac{d_{a}}{d_{b}, m}}^{(b)}(u)}{\left(1+Y_{m-1}^{(a)}(u)^{-1}\right)\left(1+Y_{m+1}^{(a)}(u)^{-1}\right)} \quad \text { if } \quad d_{a}>1, \\
& Y_{m}^{(a)}\left(u-\frac{d_{a}}{t}\right) Y_{m}^{(a)}\left(u+\frac{d_{a}}{t}\right)=\frac{\prod_{b: b \sim a}\left(1+Y_{\frac{m}{d_{b}}}^{(b)}(u)\right)}{\left(1+Y_{m-1}^{(a)}(u)^{-1}\right)\left(1+Y_{m+1}^{(a)}(u)^{-1}\right)} \quad \text { if } \quad d_{a}=1,
\end{aligned}
$$

where for $p \in \mathbb{N}$

$$
Z_{p, m}^{(b)}(u)=\prod_{j=-p+1}^{p-1}\left\{\prod_{k=1}^{p-|j|}\left(1+Y_{p m+j}^{(b)}\left(u+\frac{1}{t}(p-|j|+1-2 k)\right)\right)\right\}
$$

and $Y_{\frac{m}{d_{b}}}^{(b)}(u)=0$ in $(4.2)$ if $\frac{m}{d_{b}} \notin \mathbb{N}$.

\section{Remark 4.2.}

1. The Y-systems here are formally in the same form as the ones for the quantum affine algebras [42]. However, $p$ for $Z_{p, m}^{(b)}(u)$ here may be greater than 3 .

2. In the right hand side of (4.1), $\frac{d_{a}}{d_{b}}$ is either 1 or $d_{a}$ due to (3.1). The term $Z_{p, m}^{(b)}(u)$ is written more explicitly as follows: for $p=1$,

$$
Z_{1, m}^{(b)}(u)=1+Y_{m}^{(b)}(u)
$$

for $p=2$,

$$
Z_{2, m}^{(b)}(u)=\left(1+Y_{2 m-1}^{(b)}(u)\right)\left(1+Y_{2 m}^{(b)}\left(u-\frac{1}{t}\right)\right)\left(1+Y_{2 m}^{(b)}\left(u+\frac{1}{t}\right)\right)\left(1+Y_{2 m+1}^{(b)}(u)\right),
$$

for $p=3$,

$$
\begin{aligned}
Z_{3, m}^{(b)}(u)= & \left(1+Y_{3 m-2}^{(b)}(u)\right)\left(1+Y_{3 m-1}^{(b)}\left(u-\frac{1}{t}\right)\right)\left(1+Y_{3 m-1}^{(b)}\left(u+\frac{1}{t}\right)\right) \\
& \times\left(1+Y_{3 m}^{(b)}\left(u-\frac{2}{t}\right)\right)\left(1+Y_{3 m}^{(b)}(u)\right)\left(1+Y_{3 m}^{(b)}\left(u+\frac{2}{t}\right)\right) \\
& \times\left(1+Y_{3 m+1}^{(b)}\left(u-\frac{1}{t}\right)\right)\left(1+Y_{3 m+1}^{(b)}\left(u+\frac{1}{t}\right)\right)\left(1+Y_{3 m+2}^{(b)}(u)\right),
\end{aligned}
$$

and so on. There are $p^{2}$ factors in $Z_{p, m}^{(b)}(u)$.

\subsection{Relation between $\mathrm{T}$ and $\mathrm{Y}$-systems}

Let us write both the relations (3.2) and (3.3) in $\mathbb{T}(C)$ in a unified manner

$$
\begin{aligned}
T_{m}^{(a)}\left(u-\frac{d_{a}}{t}\right) T_{m}^{(a)}\left(u+\frac{d_{a}}{t}\right) & =T_{m-1}^{(a)}(u) T_{m+1}^{(a)}(u)+M_{m}^{(a)}(u) \\
& =T_{m-1}^{(a)}(u) T_{m+1}^{(a)}(u)+\prod_{(b, k, v)} T_{k}^{(b)}(v)^{G(b, k, v ; a, m, u)},
\end{aligned}
$$

where $M_{m}^{(a)}(u)$ is the second term of the right hand side of each relation. Define the transposition ${ }^{t} G(b, k, v ; a, m, u)=G(a, m, u ; b, k, v)$. 
Theorem 4.3. The $Y$-system $\mathbb{Y}(C)$ is written as

$$
Y_{m}^{(a)}\left(u-\frac{d_{a}}{t}\right) Y_{m}^{(a)}\left(u+\frac{d_{a}}{t}\right)=\frac{\prod_{(b, k, v)}\left(1+Y_{k}^{(b)}(v)\right)^{t} G(b, k, v ; a, m, u)}{\left(1+Y_{m-1}^{(a)}(u)^{-1}\right)\left(1+Y_{m+1}^{(a)}(u)^{-1}\right)} .
$$

Proof. This can be proved by case check for $d_{a}>1$ and $d_{a}=1$.

For any commutative ring $R$ over $\mathbb{Z}$ with identity element, let $R^{\times}$denote the group of all the invertible elements of $R$.

Theorem 4.4. Let $R$ be any commutative ring over $\mathbb{Z}$ with identity element.

(1) For any family $T=\left\{T_{m}^{(a)}(u) \in R^{\times} \mid a \in I, m \in \mathbb{N}, u \in U\right\}$ satisfying $\mathbb{T}(C)$ in $R$, define a family $Y=\left\{Y_{m}^{(a)}(u) \in R^{\times} \mid a \in I, m \in \mathbb{N}, u \in U\right\}$ by

$$
Y_{m}^{(a)}(u)=\frac{M_{m}^{(a)}(u)}{T_{m-1}^{(a)}(u) T_{m+1}^{(a)}(u)},
$$

where $T_{0}^{(a)}(u)=1$. Then,

$$
\begin{aligned}
& 1+Y_{m}^{(a)}(u)=\frac{T_{m}^{(a)}\left(u-\frac{d_{a}}{t}\right) T_{m}^{(a)}\left(u+\frac{d_{a}}{t}\right)}{T_{m-1}^{(a)}(u) T_{m+1}^{(a)}(u)}, \\
& 1+Y_{m}^{(a)}(u)^{-1}=\frac{T_{m}^{(a)}\left(u-\frac{d_{a}}{t}\right) T_{m}^{(a)}\left(u+\frac{d_{a}}{t}\right)}{M_{m}^{(a)}(u)} .
\end{aligned}
$$

Furthermore, $Y$ satisfies $\mathbb{Y}(C)$ in $R$.

(2) Conversely, for any family $Y=\left\{Y_{m}^{(a)}(u) \in R^{\times} \mid a \in I, m \in \mathbb{N}, u \in U\right\}$ satisfying $\mathbb{Y}(C)$ with $1+Y_{m}^{(a)}(u)^{ \pm 1} \in R^{\times}$, there is a (not unique) family $T=\left\{T_{m}^{(a)}(u) \in R^{\times} \mid a \in I, m \in \mathbb{N}\right.$, $u \in U\}$ satisfying $\mathbb{T}(C)$ such that $Y_{m}^{(a)}(u)$ is given by (4.4).

Proof. (1) Equations (4.5) and (4.6) follow from (4.3) and (4.4). We show that $Y$ satisfies $\mathbb{Y}(C)$. For $d_{a}>1$, by (4.4)-(4.6), the relation (4.1) reduces to the following identity: For any $p \in \mathbb{N}$,

$$
\frac{T_{p m}^{(b)}\left(u-\frac{p}{t}\right) T_{p m}^{(b)}\left(u+\frac{p}{t}\right)}{T_{p(m-1)}^{(b)}(u) T_{p(m+1)}^{(b)}(u)}=\prod_{j=-p+1}^{p-1}\left\{\prod_{k=1}^{p-|j|} \frac{T_{p m+j}^{(b)}\left(\tilde{u}-\frac{1}{t}\right) T_{p m+j}^{(b)}\left(\tilde{u}+\frac{1}{t}\right)}{T_{p m+j-1}^{(b)}(\tilde{u}) T_{p m+j+1}^{(b)}(\tilde{u})}\right\},
$$

where $\tilde{u}=u+\frac{1}{t}(p-|j|+1-2 k)$. This is easily proved without using $\mathbb{T}(C)$. Similarly, for $d_{a}=1$, the relation (4.2) reduces to the following identity:

$$
\frac{S_{m}^{(b)}\left(u-\frac{1}{t}\right) S_{m}^{(b)}\left(u+\frac{1}{t}\right)}{S_{m-1}^{(b)}(u) S_{m+1}^{(b)}(u)}= \begin{cases}\frac{T_{\frac{m}{d_{b}}}^{(b)}\left(u-\frac{d_{b}}{t}\right) T_{\frac{m}{d_{b}}}^{(b)}\left(u+\frac{d_{b}}{t}\right)}{T_{\frac{m}{d_{b}}-1}^{(b)}(u) T_{\frac{m}{d_{b}}+1}^{(b)}(u)}, & \frac{m}{d_{b}} \in \mathbb{N}, \\ 1, & \text { otherwise, }\end{cases}
$$

where $S_{m}^{(b)}(u)$ is defined in (3.4). Again, this is easily proved without using $\mathbb{T}(C)$.

(2) We modify the proof in the case of quantum affine algebras [32, Theorem 2.12] so that it is applicable to the present situation. Here, we concentrate on the case $U=\frac{1}{t} \mathbb{Z}$. The modification of the proof for the other cases $U=\mathbb{C}$ and $\mathbb{C} /(2 \pi \sqrt{-1} / \xi) \mathbb{Z}$ is straightforward.

Case 1. When $C$ is simply laced. Suppose that $C$ is simply laced. Thus, $d_{a}=1$ for any $a \in I$ and $t=1$. For any $Y$ satisfying $\mathbb{Y}(C)$, we construct a desired family $T$ in the following three steps: 
Step 1. Choose arbitrarily $T_{1}^{(a)}(-1), T_{1}^{(a)}(0), \in R^{\times}(a \in I)$.

Step 2. Define $T_{1}^{(a)}(-2), T_{1}^{(a)}(1)(a \in I)$ by

$$
T_{1}^{(a)}(u \pm 1)=\left(1+Y_{1}^{(a)}(u)^{-1}\right) \frac{M_{1}^{(a)}(u)}{T_{1}^{(a)}(u \mp 1)} .
$$

Repeat it and define $T_{1}^{(a)}(u)(a \in I)$ for the rest of $u \in \mathbb{Z}$ by (4.9).

Step 3. Define $T_{m}^{(a)}(u)(a \in I, u \in \mathbb{Z})$ for $m \geq 2$ by

$$
T_{m+1}^{(a)}(u)=\frac{1}{1+Y_{m}^{(a)}(u)} \frac{T_{m}^{(a)}(u-1) T_{m}^{(a)}(u+1)}{T_{m-1}^{(a)}(u)},
$$

where $T_{0}^{(a)}(u)=1$.

Claim. The family $T$ defined above satisfies the following relations in $R$ for any $a \in I, m \in \mathbb{N}$, $u \in \mathbb{Z}:$

$$
\begin{aligned}
& Y_{m}^{(a)}(u)=\frac{M_{m}^{(a)}(u)}{T_{m-1}^{(a)}(u) T_{m+1}^{(a)}(u)}, \\
& 1+Y_{m}^{(a)}(u)=\frac{T_{m}^{(a)}(u-1) T_{m}^{(a)}(u+1)}{T_{m-1}^{(a)}(u) T_{m+1}^{(a)}(u)}, \\
& 1+Y_{m}^{(a)}(u)^{-1}=\frac{T_{m}^{(a)}(u-1) T_{m}^{(a)}(u+1)}{M_{m}^{(a)}(u)} .
\end{aligned}
$$

Proof of Claim. (4.12) holds by (4.10). (4.11) and (4.13) are equivalent under (4.12); furthermore, (4.13) holds for $m=1$ by (4.9). So it is enough to show (4.11) for $m \geq 2$ by induction on $m$. In fact,

$$
\begin{aligned}
& \frac{M_{m}^{(a)}(u)}{T_{m-1}^{(a)}(u) T_{m+1}^{(a)}(u)} \stackrel{\text { by }}{\stackrel{(4.12)}{=}} \frac{\left(1+Y_{m-2}^{(a)}(u)\right) T_{m-3}^{(a)}(u)}{T_{m-2}^{(a)}(u-1) T_{m-2}^{(a)}(u+1)} \frac{\left(1+Y_{m}^{(a)}(u)\right) T_{m-1}^{(a)}(u)}{T_{m}^{(a)}(u-1) T_{m}^{(a)}(u+1)} M_{m}^{(a)}(u) \\
& \stackrel{\substack{\text { by induction } \\
\text { hypothesis }}}{=}\left(1+Y_{m-2}^{(a)}(u)\right)\left(1+Y_{m}^{(a)}(u)\right) \frac{Y_{m-1}^{(a)}(u-1) Y_{m-1}^{(a)}(u+1)}{Y_{m-2}^{(a)}(u)} \frac{M_{m-2}^{(a)}(u) M_{m}^{(a)}(u)}{M_{m-1}^{(a)}(u-1) M_{m-1}^{(a)}(u+1)} \\
& \text { by (4.12) and } \mathbb{Y}(C) Y_{m}^{(a)}(u) \text {. }
\end{aligned}
$$

This ends the proof of Claim.

Now, taking the inverse sum of (4.12) and (4.13), we obtain (4.3). Therefore, $T$ satisfies the desired properties.

Case 2. When $C$ is nonsimply laced. Suppose that $C$ is nonsimply laced. Then, in Step 2 above, the factor $M_{1}^{(a)}(u)$ in (4.9) involves the term $T_{d_{a}}^{(b)}(u)$ for $a$ and $b$ with $a \sim b, d_{a}>1$, and $d_{b}=1$. Therefore, Step 2 should be modified to define these terms together. For any $Y$ satisfying $\mathbb{Y}(C)$, we construct a desired family $T$ in the following three steps:

Step 1. Choose arbitrarily $T_{1}^{(a)}(u) \in R^{\times}\left(a \in I,-\frac{d_{a}}{t} \leq u<\frac{d_{a}}{t}\right)$.

Step 2. Define $T_{1}^{(a)}(u)(a \in I)$ for the rest of $u \in \frac{1}{t} \mathbb{Z}$ as below. (One can easily check that each step is well-defined.)

Let $\left\{1<p_{1}<p_{2}<\cdots<p_{k}\right\}=\left\{d_{a} \mid a \in I\right\}$, and $I=I_{1} \sqcup I_{p_{1}} \sqcup I_{p_{2}} \sqcup \cdots \sqcup I_{p_{k}}$, where $I_{p}:=\left\{a \in I \mid d_{a}=p\right\}$. 
Substep 1.

$(i)_{1}$. Define $T_{1}^{(a)}\left(-\frac{2}{t}\right), T_{1}^{(a)}\left(\frac{1}{t}\right)\left(a \in I_{1}\right)$ by

$$
T_{1}^{(a)}\left(u \pm \frac{d_{a}}{t}\right)=\left(1+Y_{1}^{(a)}(u)^{-1}\right) \frac{M_{1}^{(a)}(u)}{T_{1}^{(a)}\left(u \mp \frac{d_{a}}{t}\right)} .
$$

$(i i)_{1}$. Define $T_{1}^{(a)}(u)\left(a \in I_{1}\right)$ for the rest of $-\frac{p_{1}}{t} \leq u<\frac{p_{1}}{t}$ by repeating $(i)_{1}$.

Substep 2.

$(i)_{2}$. Define $T_{p_{1}}^{(a)}\left(-\frac{1}{t}\right), T_{p_{1}}^{(a)}(0)\left(a \in I_{1}\right)$ by

$$
T_{m+1}^{(a)}(u)=\frac{1}{1+Y_{m}^{(a)}(u)} \frac{T_{m}^{(a)}\left(u-\frac{d_{a}}{t}\right) T_{m}^{(a)}\left(u+\frac{d_{a}}{t}\right)}{T_{m-1}^{(a)}(u)},
$$

where $T_{0}^{(a)}(u)=1$.

$(\text { ii })_{2}$. Define $T_{1}^{(a)}\left(-\frac{p_{1}}{t}-\frac{1}{t}\right), T_{1}^{(a)}\left(\frac{p_{1}}{t}\right)\left(a \in I_{1} \sqcup I_{p_{1}}\right)$ by (4.14).

$(\text { iii })_{2}$. Define $T_{1}^{(a)}(u)\left(a \in I_{1} \sqcup I_{p_{1}}\right)$ for the rest of $-\frac{p_{2}}{t} \leq u<\frac{p_{2}}{t}$ by repeating $(i)_{2}-(i i)_{2}$.

\section{Substep 3.}

$(i)_{3}$. Define $T_{p_{1}}^{(a)}\left(-\frac{p_{2}}{t}+\frac{p_{1}}{t}-\frac{1}{t}\right), T_{p_{1}}^{(a)}\left(\frac{p_{2}}{t}-\frac{p_{1}}{t}\right)\left(a \in I_{1}\right)$ by (4.15).

Define $T_{p_{2}}^{(a)}\left(-\frac{1}{t}\right), T_{p_{2}}^{(a)}(0)\left(a \in I_{1}\right)$ by $(4.15)$.

$(i i)_{3}$. Define $T_{1}^{(a)}\left(-\frac{p_{2}}{t}-\frac{1}{t}\right), T_{1}^{(a)}\left(\frac{p_{2}}{t}\right)\left(a \in I_{1} \sqcup I_{p_{1}} \sqcup I_{p_{2}}\right)$ by (4.14).

$(i i i)_{3}$. Define $T_{1}^{(a)}(u)\left(a \in I_{1} \sqcup I_{p_{1}} \sqcup I_{p_{2}}\right)$ for the rest of $-\frac{p_{3}}{t} \leq u<\frac{p_{3}}{t}$ by repeating $(i)_{3}-(i i)_{3}$.

Substep 4. Continue to define $T_{1}^{(a)}(u)(a \in I)$ for the rest of $u \in \frac{1}{t} \mathbb{Z}$.

Step 3. Define $T_{m}^{(a)}(u)\left(a \in I, u \in \frac{1}{t} \mathbb{Z}\right)$ for $m \geq 2$ by (4.15).

Notice that $T_{1}^{(a)}(u)$ is always defined by (4.14), while $T_{m}^{(a)}(u)$ for $m \geq 2$ is so by (4.15). Then, the rest of the proof can be done in a parallel way to the simply laced case by using (4.7) and (4.8).

Definition 4.5. Let $y(C)$ be the commutative ring over $\mathbb{Z}$ with generators $Y_{m}^{(a)}(u)^{ \pm 1}$, $\left(1+Y_{m}^{(a)}(u)\right)^{-1}(a \in I, m \in \mathbb{N}, u \in U)$ and the relations $\mathbb{Y}(C)$.

In terms of $\mathcal{T}(C)$ and $y(C)$, Theorem 4.4 is rephrased as follows (cf. [32, Theorem 2.12] for the quantum affine algebras):

\section{Theorem 4.6.}

(1) There is a ring homomorphism

$$
\varphi: y(C) \rightarrow \mathcal{T}(C)
$$

defined by

$$
Y_{m}^{(a)}(u) \mapsto \frac{M_{m}^{(a)}(u)}{T_{m-1}^{(a)}(u) T_{m+1}^{(a)}(u)} .
$$

(2) There is a (not unique) ring homomorphism

$$
\psi: \mathcal{T}(C) \rightarrow y(C)
$$

such that $\psi \circ \varphi=\mathrm{idy}_{(C)}$. 
There is another variation of Theorem 4.4. Let $\mathcal{T}^{\times}(C)\left(\right.$ resp. $\left.y^{\times}(C)\right)$ be the multiplicative subgroup of all the invertible elements of $\mathcal{T}(C)$ (resp. $y(C)$ ). Clearly, $\mathcal{T}^{\times}(C)$ is generated by $T_{m}^{(a)}(u)$ 's, while $y^{\times}(C)$ is generated by $Y_{m}^{(a)}(u)$ 's and $1+Y_{m}^{(a)}(u)$ 's.

\section{Theorem 4.7.}

(1) There is a multiplicative group homomorphism

$$
\varphi: y^{\times}(C) \rightarrow \mathcal{T}^{\times}(C)
$$

defined by

$$
\begin{aligned}
& Y_{m}^{(a)}(u) \mapsto \frac{M_{m}^{(a)}(u)}{T_{m-1}^{(a)}(u) T_{m+1}^{(a)}(u)}, \\
& 1+Y_{m}^{(a)}(u) \mapsto \frac{T_{m}^{(a)}\left(u-\frac{d_{a}}{t}\right) T_{m}^{(a)}\left(u+\frac{d_{a}}{t}\right)}{T_{m-1}^{(a)}(u) T_{m+1}^{(a)}(u)} .
\end{aligned}
$$

(2) There is a (not unique) multiplicative group homomorphism

$$
\psi: \mathcal{T}^{\times}(C) \rightarrow y^{\times}(C)
$$

such that $\psi \circ \varphi=\operatorname{id}_{y(C)} \times$.

\section{$5 \quad$ Restricted T and Y-systems}

Here we introduce a series of reductions of the systems $\mathbb{T}(C)$ and $\mathbb{Y}(C)$ called the restricted $\mathrm{T}$ and $\mathrm{Y}$-systems. The restricted $\mathrm{T}$ and $\mathrm{Y}$-systems for the quantum affine algebras are important in application to various integrable models.

We define integers $t_{a}(a \in I)$ by

$$
t_{a}=\frac{t}{d_{a}}
$$

Definition 5.1. Fix an integer $\ell \geq 2$. For a tamely laced generalized Cartan matrix $C$, the level $\ell$ restricted T-system $\mathbb{T}_{\ell}(C)$ associated with $C$ (with the unit boundary condition) is the system of relations (3.2) and (3.3) naturally restricted to a family of variables $T_{\ell}=\left\{T_{m}^{(a)}(u) \mid\right.$ $\left.a \in I ; m=1, \ldots, t_{a} \ell-1 ; u \in U\right\}$, where $T_{0}^{(a)}(u)=1$, and furthermore, $T_{t_{a} \ell}^{(a)}(u)=1$ (the unit boundary condition) if they occur in the right hand sides in the relations.

Definition 5.2. Fix an integer $\ell \geq 2$. For a tamely laced generalized Cartan matrix $C$, the level $\ell$ restricted $Y$-system $\mathbb{Y}_{\ell}(C)$ associated with $C$ is the system of relations (4.1) and (4.2) naturally restricted to a family of variables $Y_{\ell}=\left\{Y_{m}^{(a)}(u) \mid a \in I ; m=1, \ldots, t_{a} \ell-1 ; u \in U\right\}$, where $Y_{0}^{(a)}(u)^{-1}=0$, and furthermore, $Y_{t_{a} \ell}^{(a)}(u)^{-1}=0$ if they occur in the right hand sides in the relations.

The restricted version of Theorem 4.4 (1) holds.

Theorem 5.3. Let $R$ be any commutative ring over $\mathbb{Z}$ with identity element. For any family $T_{\ell}=\left\{T_{m}^{(a)}(u) \in R^{\times} \mid a \in I ; m=1, \ldots, t_{a} \ell-1 ; u \in U\right\}$ satisfying $\mathbb{T}_{\ell}(C)$ in $R$, define a family $Y_{\ell}=\left\{Y_{m}^{(a)}(u) \in R^{\times} \mid a \in I ; m=1, \ldots, t_{a} \ell-1 ; u \in U\right\}$ by $(4.4)$, where $T_{0}^{(a)}(u)=T_{t_{a} \ell}^{(a)}(u)=1$. Then, $Y_{\ell}$ satisfies $\mathbb{Y}_{\ell}(C)$ in $R$. 
Proof. The calculation is formally the same as the one for Theorem 4.4. We have only to take care of the boundary term

$$
\frac{1}{1+Y_{t_{a} \ell}^{(a)}(u)^{-1}}=\frac{M_{t_{a} \ell}^{(a)}(u)}{T_{t_{a} \ell}^{(a)}\left(u-\frac{d_{a}}{t}\right) T_{t_{a} \ell}^{(a)}\left(u+\frac{d_{a}}{t}\right)},
$$

which formally appears in the right hand sides of (4.1) and (4.2) for $m=t_{a} \ell-1$. Since

$$
M_{t_{a} \ell}^{(a)}(u)= \begin{cases}\prod_{b: b \sim a} T_{t_{b} \ell}^{(b)}(u), & d_{a}>1, \\ \prod_{b: b \sim a}\left\{\prod_{k=1}^{d_{b}} T_{t_{b} \ell}^{(b)}\left(u+\frac{1}{t}\left(2 k-1-d_{b}\right)\right)\right\}, & d_{a}=1,\end{cases}
$$

the right hand side of (5.1) is 1 under the boundary condition $T_{t_{a} \ell}^{(a)}(u)=1$ of $\mathbb{T}_{\ell}(C)$. This is compatible with $Y_{t_{a} \ell}^{(a)}(u)^{-1}=0$.

Unfortunately the restricted version of Theorem 4.4 (2) does not hold due to the boundary condition of $\mathbb{T}_{\ell}(C)$.

\section{$6 \quad \mathrm{~T}$ and $\mathrm{Y}$-systems from cluster algebras}

In this section we introduce $\mathrm{T}$ and $\mathrm{Y}$-systems associated with a class of cluster algebras [17, 19] by generalizing some of the results in $[19,29,10,36,32,11]$. They include the restricted $\mathrm{T}$ and Y-systems of simply laced type in Section 5 as special cases.

\subsection{Systems $\mathbb{T}(B)$ and $\mathbb{Y}_{ \pm}(B)$}

We warn the reader that the matrix $B$ in this section is different from the one in Section 2 and should not be confused.

Definition 6.1 ([16]). An integer matrix $B=\left(B_{i j}\right)_{i, j \in I}$ is skew-symmetrizable if there is a diagonal matrix $D=\operatorname{diag}\left(d_{i}\right)_{i \in I}$ with $d_{i} \in \mathbb{N}$ such that $D B$ is skew-symmetric. For a skewsymmetrizable matrix $B$ and $k \in I$, another matrix $B^{\prime}=\mu_{k}(B)$, called the mutation of $B$ at $k$, is defined by

$$
B_{i j}^{\prime}= \begin{cases}-B_{i j}, & i=k \text { or } j=k, \\ B_{i j}+\frac{1}{2}\left(\left|B_{i k}\right| B_{k j}+B_{i k}\left|B_{k j}\right|\right), & \text { otherwise. }\end{cases}
$$

The matrix $\mu_{k}(B)$ is also skew-symmetrizable. The matrix mutation plays a central role in the theory of cluster algebras.

We impose the following conditions on a skew-symmetrizable matrix $B$ : The index set $I$ admits the decomposition $I=I_{+} \sqcup I_{-}$such that

$$
\text { if } B_{i j} \neq 0 \text {, then }(i, j) \in I_{+} \times I_{-} \text {or }(i, j) \in I_{-} \times I_{+} \text {. }
$$

Furthermore, for composed mutations $\mu_{+}=\prod_{i \in I_{+}} \mu_{i}$ and $\mu_{-}=\prod_{i \in I_{-}} \mu_{i}$,

$$
\mu_{+}(B)=\mu_{-}(B)=-B .
$$

Note that $\mu_{ \pm}(B)$ does not depend on the order of the product due to (6.2). 
Lemma 6.2. Under the condition (6.2), the condition (6.3) is equivalent to the following one: For any $i, j \in I_{+}$,

$$
\sum_{k: B_{i k}>0, B_{k j}>0} B_{i k} B_{k j}=\sum_{k: B_{i k}<0, B_{k j}<0} B_{i k} B_{k j} .
$$

The same holds for $i, j \in I_{-}$.

Proof. Suppose that (6.3) holds. Then, for any $i, j \in I_{+}, \mu_{-}(B)_{i j}=-B_{i j}=0$ by (6.2). It follows from (6.1) that

$$
\sum_{k: B_{i k} B_{k j}>0}\left|B_{i k}\right| B_{k j}=0 .
$$

Therefore, we have (6.4). The rest of the proof is similar.

Definition 6.3. For a skew-symmetrizable matrix $B$ satisfying the conditions (6.2) and (6.3), the T-system $\mathbb{T}(B)$ associated with $B$ is the following system of relations for a family of variables $T=\left\{T_{i}(u) \mid i \in I, u \in \mathbb{Z}\right\}:$

$$
T_{i}(u-1) T_{i}(u+1)=\prod_{j: B_{j i}>0} T_{j}(u)^{B_{j i}}+\prod_{j: B_{j i}<0} T_{j}(u)^{-B_{j i}} .
$$

For Y-systems, it is natural to introduce two kinds of systems.

Definition 6.4. For a skew-symmetrizable matrix $B$ satisfying the conditions (6.2) and (6.3), the $Y$-systems $\mathbb{Y}_{+}(B)$ and $\mathbb{Y}_{-}(B)$ associated with $B$ are the following systems of relations for a family of variables $Y=\left\{Y_{i}(u) \mid i \in I, u \in \mathbb{Z}\right\}$, respectively: For $\mathbb{Y}_{+}(B)$,

$$
Y_{i}(u-1) Y_{i}(u+1)=\frac{\prod_{j: B_{j i} \gtrless 0}\left(1+Y_{j}(u)\right)^{ \pm B_{j i}}}{\prod_{j: B_{j i} \lessgtr 0}\left(1+Y_{j}(u)^{-1}\right)^{\mp B_{j i}}}, \quad i \in I_{ \pm} .
$$

For $\mathbb{Y}_{-}(B)$,

$$
Y_{i}(u-1) Y_{i}(u+1)=\frac{\prod_{j: B_{j i} \lessgtr 0}\left(1+Y_{j}(u)\right)^{\mp B_{j i}}}{\prod_{j: B_{j i} \gtrless 0}\left(1+Y_{j}(u)^{-1}\right)^{ \pm B_{j i}}}, \quad i \in I_{ \pm} .
$$

Remark 6.5. Two systems, $\mathbb{Y}_{+}(B)$ and $\mathbb{Y}_{-}(B)$, are transformed into each other by any of the exchanges, $Y_{i}(u) \leftrightarrow Y_{i}(u)^{-1}, I_{ \pm} \leftrightarrow I_{\mp}$, or $B \leftrightarrow-B$. There is no preferred choice between $\mathbb{Y}_{+}(B)$ and $\mathbb{Y}_{-}(B)$, a priori, and a convenient one can be used depending on the context. On the contrary, $\mathbb{T}(B)$ is invariant under either $I_{ \pm} \leftrightarrow I_{\mp}$ or $B \leftrightarrow-B$.

Theorem 6.6. Let $R$ be any commutative ring over $\mathbb{Z}$ with identity element. For any family $T=\left\{T_{i}(u) \in R^{\times} \mid i \in I, u \in \mathbb{Z}\right\}$ satisfying $\mathbb{T}(B)$ in $R$, define a family $Y=\left\{Y_{i}(u) \in R^{\times} \mid i \in I\right.$, $u \in \mathbb{Z}\}$ by

$$
Y_{i}(u)=\prod_{j \in I} T_{j}(u)^{ \pm B_{j i}}, \quad i \in I_{ \pm}
$$

Then, $Y$ satisfies $\mathbb{Y}_{+}(B)$. Similarly, define a family $Y$ by

$$
Y_{i}(u)=\prod_{j \in I} T_{j}(u)^{\mp B_{j i}}, \quad i \in I_{ \pm} .
$$

Then, $Y$ satisfies $\mathbb{Y}_{-}(B)$ in $R$. 
Proof. By Remark 6.5, it is enough to prove the first statement only. Then,

$$
\begin{gathered}
\prod_{j} T_{j}(u)^{ \pm B_{j i}} \\
Y_{j: B_{j i} \gtrless 0} T_{j}(u)^{\mp B_{j i}}, \quad i \in I_{ \pm}, \\
1+Y_{i}(u)=\frac{T_{i}(u-1) T_{i}(u+1)}{\prod_{j: B_{j i} \lessgtr 0} T_{j}(u)^{\mp B_{j i}}}, \quad i \in I_{ \pm}, \\
1+Y_{i}(u)^{-1}=\frac{T_{i}(u-1) T_{i}(u+1)}{\prod_{j: B_{j i} \gtrless 0} T_{j}(u)^{ \pm B_{j i}}}, \quad i \in I_{ \pm} .
\end{gathered}
$$

Note that for $j$ in the right hand side of (6.5), $j \in I_{\mp}$ by (6.2). By putting (6.6)-(6.8) into (6.5), the right hand side of $(6.5)$ is

$$
\begin{aligned}
\prod_{j \in I}\left\{T_{j}(u-1) T_{j}(u+1)\right\}^{ \pm B_{j i}} \prod_{j: B_{j i} \gtrless 0}\left\{\prod_{k: B_{k j} \gtrless 0} T_{k}(u)^{\mp B_{k j}}\right\}^{ \pm B_{j i}} \prod_{j: B_{j i} \lessgtr 0}\left\{\prod_{k: B_{k j} \lessgtr 0} T_{k}(u)^{ \pm B_{k j}}\right\}^{ \pm B_{j i}} \\
\stackrel{(6.4)}{=} \prod_{j \in I}\left\{T_{j}(u-1) T_{j}(u+1)\right\}^{ \pm B_{j i}},
\end{aligned}
$$

which is the left hand side of (6.5).

\subsection{Examples}

Let us present some examples of $\mathbb{T}(B)$ and $\mathbb{Y}_{ \pm}(B)$.

Definition 6.7. A symmetrizable generalized Cartan matrix $C=\left(C_{i j}\right)_{i, j \in I}$ is said to be bipartite if the index set $I$ admits the decomposition $I=I_{+} \sqcup I_{-}$such that

$$
\text { if } C_{i j}<0 \text {, then }(i, j) \in I_{+} \times I_{-} \text {or }(i, j) \in I_{-} \times I_{+} \text {. }
$$

Example $6.8([17,19])$. Let $C$ be a bipartite symmetrizable generalized Cartan matrix, which is not necessarily tamely laced. Define the matrix $B=B(C)$ by

$$
B_{i j}= \begin{cases}-C_{i j}, & (i, j) \in I_{+} \times I_{-}, \\ C_{i j}, & (i, j) \in I_{-} \times I_{+}, \\ 0, & \text { otherwise. }\end{cases}
$$

The rule (6.9) is visualized in the diagram:

$$
+\stackrel{-C}{\rightarrow}-
$$

Then, $B$ is skew-symmetrizable and satisfies the conditions (6.2) and (6.3). The corresponding $\mathbb{T}(B)$ and $\mathbb{Y}_{-}(B)$ are given by

$$
\begin{aligned}
& T_{i}(u-1) T_{i}(u+1)=1+\prod_{j: j \sim i} T_{j}(u)^{-C_{j i}}, \\
& Y_{i}(u-1) Y_{i}(u+1)=\prod_{j: j \sim i}\left(1+Y_{j}(u)\right)^{-C_{j i}},
\end{aligned}
$$

where $j \sim i$ means $C_{j i}<0$. These systems are studied in [17, 19]. When $C$ is bipartite and simply laced, they coincide with $\mathbb{T}_{2}(C)$ and $\mathbb{Y}_{2}(C)$ (for $U=\mathbb{Z}$ ) in Section 5 . When $C$ is bipartite, tamely laced, but nonsimply laced, they are different from $\mathbb{T}_{2}(C)$ and $\mathbb{Y}_{2}(C)$, because the latter include factors depending on $u+\alpha(\alpha \neq 0)$ in the right hand sides. 
Example 6.9 (Square product $[29,10,36,32,11]$ ). Let $C=\left(C_{i j}\right)_{i, j \in I}$ and $C^{\prime}=$ $\left(C_{i^{\prime} j^{\prime}}^{\prime}\right)_{i^{\prime}, j^{\prime} \in I^{\prime}}$ be a pair of bipartite symmetrizable generalized Cartan matrices with $I=I_{+} \sqcup I_{-}$ and $I^{\prime}=I_{+}^{\prime} \sqcup I_{-}^{\prime}$, which are not necessarily tamely laced. For $\mathbf{i}=\left(i, i^{\prime}\right) \in I \times I^{\prime}$, let us write $\mathbf{i}:(++)$ if $\left(i, i^{\prime}\right) \in I_{+} \times I_{+}^{\prime}$, etc. Define the matrix $B=\left(B_{\mathbf{i j}}\right)_{\mathbf{i}, \mathbf{j} \in I \times I^{\prime}}$ by

$$
B_{\mathbf{i j}}= \begin{cases}-C_{i j} \delta_{i^{\prime} j^{\prime}}, & \mathbf{i}:(-+), \mathbf{j}:(++) \text { or } \mathbf{i}:(+-), \mathbf{j}:(--), \\ C_{i j} \delta_{i^{\prime} j^{\prime}}, & \mathbf{i}:(++), \mathbf{j}:(-+) \text { or } \mathbf{i}:(--), \mathbf{j}:(+-), \\ -\delta_{i j} C_{i^{\prime} j^{\prime}}^{\prime}, & \mathbf{i}:(++), \mathbf{j}:(+-) \text { or } \mathbf{i}:(--), \mathbf{j}:(-+), \\ \delta_{i j} C_{i^{\prime} j^{\prime}}^{\prime}, & \mathbf{i}:(+-), \mathbf{j}:(++) \text { or } \mathbf{i}:(-+), \mathbf{j}:(--), \\ 0, & \text { otherwise. }\end{cases}
$$

The rule (6.10) is visualized in the diagram:

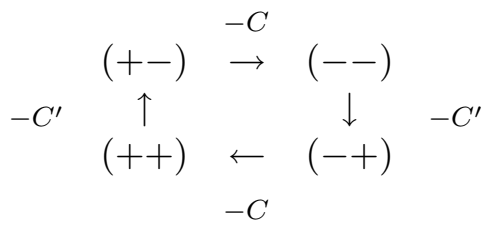

Since it generalizes the square product of quivers by [36], we call the matrix $B$ the square product $B(C) \square B\left(C^{\prime}\right)$ of the matrices $B(C)$ and $B\left(C^{\prime}\right)$ of $(6.9)$.

Lemma 6.10. The matrix $B$ in (6.10) is skew-symmetrizable and satisfies the conditions (6.2) and (6.3) for $\left(I \times I^{\prime}\right)_{+}:=\left(I_{+} \times I_{+}^{\prime}\right) \sqcup\left(I_{-} \times I_{-}^{\prime}\right)$ and $\left(I \times I^{\prime}\right)_{-}:=\left(I_{+} \times I_{-}^{\prime}\right) \sqcup\left(I_{-} \times I_{+}^{\prime}\right)$.

Proof. Let $\operatorname{diag}\left(d_{i}\right)_{i \in I}$ and $\operatorname{diag}\left(d_{i}^{\prime}\right)_{i \in I^{\prime}}$ be the diagonal matrices skew-symmetrizing $C$ and $C^{\prime}$, respectively, and let $D=\operatorname{diag}\left(d_{i} d_{i^{\prime}}^{\prime}\right)_{\left(i, i^{\prime}\right) \in I \times I^{\prime}}$. Then, the matrix $D B$ is skew-symmetric. The condition (6.2) is clear from (6.11). To show (6.4), suppose, for example, that $\mathbf{i}=\left(i, i^{\prime}\right):(++)$ and $\mathbf{j}=\left(j, j^{\prime}\right):(--)$. Then, $B_{\mathbf{i k}} B_{\mathbf{k j}} \neq 0$ only for $\mathbf{k}=\left(i, j^{\prime}\right)$ or $\mathbf{k}=\left(j, i^{\prime}\right)$; furthermore, $B_{\mathbf{i k}}, B_{\mathbf{k j}} \geq 0($ resp. $\leq 0)$ for $\mathbf{k}=\left(i, j^{\prime}\right)\left(\right.$ resp. $\left.\mathbf{k}=\left(j, i^{\prime}\right)\right)$, and $B_{\mathbf{i k}} B_{\mathbf{k j}}=C_{i j} C_{i^{\prime} j^{\prime}}^{\prime}$ for both. Thus, (6.4) holds. The other cases are similar.

The corresponding $\mathbb{T}(B)$ and $\mathbb{Y}_{+}(B)$ are given by

$$
\begin{aligned}
& T_{i i^{\prime}}(u-1) T_{i i^{\prime}}(u+1)=\prod_{j: j \sim i} T_{j i^{\prime}}(u)^{-C_{j i}}+\prod_{j^{\prime}: j^{\prime} \sim i^{\prime}} T_{i j^{\prime}}(u)^{-C_{j^{\prime} i^{\prime}}^{\prime},} \\
& Y_{i i^{\prime}}(u-1) Y_{i i^{\prime}}(u+1)=\frac{\prod_{j: j \sim i}\left(1+Y_{j i^{\prime}}(u)\right)^{-C_{j i}}}{\prod_{j^{\prime}: j^{\prime} \sim i^{\prime}}\left(1+Y_{i j^{\prime}}(u)^{-1}\right)^{-C_{j^{\prime} i^{\prime}}^{\prime}},}
\end{aligned}
$$

where $j \sim i$ and $j^{\prime} \sim i^{\prime}$ means $C_{j i}<0$ and $C_{j^{\prime} i^{\prime}}^{\prime}<0$, respectively. These systems slightly generalize the ones studied in connection with cluster algebras $[29,10,36,32,11]$. When $C$ is bipartite and simply laced, and $C^{\prime}$ is the Cartan matrix of type $A_{\ell-1}$ with $I_{+}^{\prime}=\{1,3, \ldots\}$ and $I_{-}^{\prime}=\{2,4, \ldots\}, \mathbb{T}(B)$ and $\mathbb{Y}_{+}(B)$ coincide with $\mathbb{T}_{\ell}(C)$ and $\mathbb{Y}_{\ell}(C)$ in Section 5 . (The choice of $I_{ \pm}^{\prime}$ is not essential here.) As in Example 6.8, when $C$ is bipartite, tamely laced, but nonsimply laced, and $C^{\prime}$ is the Cartan matrix of type $A_{\ell-1}$, they are different from $\mathbb{T}_{\ell}(C)$ and $\mathbb{Y}_{\ell}(C)$.

Example 6.11. Let us give an example which does not belong to the classes in Examples 6.8 and 6.9. Let $B=\left(B_{i j}\right)_{i, j \in I}$ with $I=\{1, \ldots, 7\}$ be the skew-symmetric matrix whose positive components are given by

$$
B_{21}=B_{13}=2, \quad B_{34}=B_{35}=B_{36}=B_{37}=B_{42}=B_{52}=B_{62}=B_{72}=1 .
$$


The matrix $B$ is represented by the following quiver:

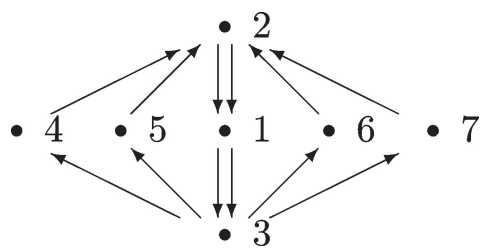

With $I_{+}=\{2,3\}$ and $I_{-}=\{1,4,5,6,7\}$, the matrix $B$ satisfies the conditions (6.2) and (6.3).

\section{3 $\mathbb{T}(B)$ and $\mathbb{Y}_{ \pm}(B)$ as relations in cluster algebras}

The systems $\mathbb{T}(B)$ and $\mathbb{Y}_{ \pm}(B)$ arise as relations for cluster variables and coefficients, respectively, in the cluster algebra associated with $B$. See $[19,36]$ for definitions and information for cluster algebras.

\subsection{1 $\mathbb{T}(B)$ and cluster algebras}

We start from T-systems.

Definition 6.12. For a skew-symmetrizable matrix $B$ satisfying the conditions (6.2) and (6.3), let $\mathcal{T}(B)$ be the commutative ring over $\mathbb{Z}$ with generators $T_{i}(u)^{ \pm 1}(i \in I, u \in \mathbb{Z})$ and the relations $\mathbb{T}(B)$. Also, let $\mathcal{T}^{\circ}(B)$ be the subring of $\mathcal{T}(B)$ generated by $T_{i}(u)(i \in I, u \in \mathbb{Z})$.

Let $\varepsilon: I \rightarrow\{+,-\}$ be the sign function defined by $\varepsilon(i)=\varepsilon$ for $i \in I_{\varepsilon}$. For $(i, u) \in I \times \mathbb{Z}$, we set the 'parity conditions' $\mathbf{P}_{+}$and $\mathbf{P}_{-}$by

$$
\mathbf{P}_{ \pm}: \varepsilon(i)(-1)^{u}= \pm
$$

where we identify + and - with 1 and -1 , respectively. For $\varepsilon \in\{+,-\}$, define $\mathcal{T}^{\circ}(B)_{\varepsilon}$ to be the subring of $\mathcal{T}^{\circ}(B)$ generated by those $T_{i}(u)$ with $(i, u)$ satisfying $\mathbf{P}_{\varepsilon}$. Then, we have $\mathcal{T}^{\circ}(B)_{+} \simeq \mathcal{T}^{\circ}(B)_{-}$by $T_{i}(u) \mapsto T_{i}(u+1)$ and

$$
\mathcal{T}^{\circ}(B) \simeq \mathcal{T}^{\circ}(B)_{+} \otimes_{\mathbb{Z}} \mathcal{T}^{\circ}(B)_{-} .
$$

Let $\mathcal{A}(B, x)$ be the cluster algebra with trivial coefficients, where $(B, x)$ is the initial seed [19]. We set $x(0)=x$ and define clusters $x(u)=\left(x_{i}(u)\right)_{i \in I}(u \in \mathbb{Z})$ by the sequence of mutations

$$
\cdots \stackrel{\mu_{-}}{\longleftrightarrow}(B, x(0)) \stackrel{\mu_{+}}{\longleftrightarrow}(-B, x(1)) \stackrel{\mu_{-}}{\longleftrightarrow}(B, x(2)) \stackrel{\mu_{+}}{\longleftrightarrow} \cdots .
$$

Definition 6.13. The T-subalgebra $\mathcal{A}_{T}(B, x)$ of $\mathcal{A}(B, x)$ associated with the sequence (6.12) is the subring of $\mathcal{A}(B, x)$ generated by $x_{i}(u)(i \in I, u \in \mathbb{Z})$.

The ring $\mathcal{A}_{T}(B, x)$ is no longer a cluster algebra in general, because it is not closed under mutations.

\section{Lemma 6.14.}

(1) $x_{i}(u)=x_{i}(u \mp 1)$ for $(i, u)$ satisfying $\mathbf{P}_{ \pm}$.

(2) The family $x=\left\{x_{i}(u) \mid i \in I, u \in \mathbb{Z}\right\}$ satisfies the T-system $\mathbb{T}(B)$ by replacing $T_{i}(u)$ in $\mathbb{T}(B)$ with $x_{i}(u)$.

Proof. This follows from the exchange relation of a cluster $x$ by the mutation $\mu_{k}$ [19]:

$$
x_{i}^{\prime}= \begin{cases}x_{i}, & i \neq k, \\ \frac{1}{x_{k}}\left(\prod_{j: B_{j i}>0} x^{B_{j i}}+\prod_{j: B_{j i}<0} x^{-B_{j i}}\right), & i=k .\end{cases}
$$


Theorem 6.15 (cf. [32, Proposition 4.24]). For $\varepsilon \in\{+,-\}$, the ring $\mathcal{T}^{\circ}(B)_{\varepsilon}$ is isomorphic to $\mathcal{A}_{T}(B, x)$ by the correspondence $T_{i}(u) \rightarrow x_{i}(u)$ for $(i, u)$ satisfying $\mathbf{P}_{\varepsilon}$.

Proof. This follows from Lemma 6.14 using the same argument as the one for [32, Proposition 4.2].

\subsection{2 $\mathbb{Y}_{\varepsilon}(B)$ and cluster algebras}

We present a parallel result for Y-systems.

A semifield $(\mathbb{P},+)$ is an abelian multiplicative group $\mathbb{P}$ endowed with a binary operation of addition + which is commutative, associative, and distributive with respect to the multiplication in $\mathbb{P}[19,31]$. (Here we use the symbol + instead of $\oplus$ in [19] to make the description a little simpler.)

Definition 6.16. For $\varepsilon \in\{+,-\}$ and a skew-symmetrizable matrix $B$ satisfying the conditions (6.2) and (6.3), let $\tilde{y}_{\varepsilon}(B)$ be the semifield with generators $Y_{i}(u)(i \in I, u \in \mathbb{Z})$ and the relations $\mathbb{Y}_{\varepsilon}(B)$. Let $\tilde{y}_{\varepsilon}^{\circ}(B)$ be the multiplicative subgroup of $\tilde{y}_{\varepsilon}(B)$ generated by $Y_{i}(u)$ and $1+Y_{i}(u)(i \in I, u \in \mathbb{Z})$. (We use the notation $\tilde{y}$ to distinguish it from the ring $y$ in Definition 4.5.)

Define $\tilde{y}_{\varepsilon}^{\circ}(B)_{+}\left(\operatorname{resp} . \tilde{y}_{\varepsilon}^{\circ}(B)_{-}\right)$to be the subgroup of $\tilde{y}_{\varepsilon}^{\circ}(B)$ generated by those $Y_{i}(u)$ and $1+Y_{i}(u)$ with $(i, u)$ satisfying $\mathbf{P}_{+}\left(\operatorname{resp} . \mathbf{P}_{-}\right)$. Then, we have $\tilde{y}_{\varepsilon}^{\circ}(B)_{+} \simeq \tilde{y}_{\varepsilon}^{\circ}(B)_{-}$by $Y_{i}(u) \mapsto$ $Y_{i}(u+1)$ and

$$
\tilde{y}_{\varepsilon}^{\circ}(B) \simeq \tilde{y}_{\varepsilon}^{\circ}(B)_{+} \times \tilde{y}_{\varepsilon}^{\circ}(B)_{-} .
$$

Let $\mathcal{A}(B, x, y)$ be the cluster algebra with coefficients in the universal semifield $\mathbb{Q}_{\mathrm{sf}}(y)$, where $(B, x, y)$ is the initial seed [19]. To make the setting parallel to T-systems, we introduce the coefficient group $\mathcal{G}(B, y)$ associated with $\mathcal{A}(B, x, y)$, which is the multiplicative subgroup of the semifield $\mathbb{Q}_{\mathrm{sf}}(y)$ generated by all the elements $y_{i}^{\prime}$ of coefficient tuples of $\mathcal{A}(B, x, y)$ together with $1+y_{i}^{\prime}$.

We set $x(0)=x, y(0)=y$ and define clusters $x(u)=\left(x_{i}(u)\right)_{i \in I}$ and coefficient tuples $y(u)=\left(y_{i}(u)\right)_{i \in I}(u \in \mathbb{Z})$ by the sequence of mutations

$$
\cdots \stackrel{\mu_{-}}{\longleftrightarrow}(B, x(0), y(0)) \stackrel{\mu_{+}}{\longleftrightarrow}(-B, x(1), y(1)) \stackrel{\mu_{-}}{\longleftrightarrow}(B, x(2), y(2)) \stackrel{\mu_{+}}{\longleftrightarrow} \cdots .
$$

Definition 6.17. The $Y$-subgroup $\mathcal{G}_{Y}(B, y)$ of $\mathcal{G}(B, y)$ associated with the sequence $(6.13)$ is the multiplicative subgroup of $\mathcal{G}(B, y)$ generated by $y_{i}(u)$ and $1+y_{i}(u)(i \in I, u \in \mathbb{Z})$.

\section{Lemma 6.18.}

(1) $y_{i}(u)=y_{i}(u \pm 1)^{-1}$ for $(i, u)$ satisfying $\mathbf{P}_{ \pm}$.

(2) For $\varepsilon \in\{+,-\}$, the family $y_{\varepsilon}=\left\{y_{i}(u) \mid(i, u)\right.$ satisfying $\left.\mathbf{P}_{\varepsilon}\right\}$ satisfies the $Y$-system $\mathbb{Y}_{\varepsilon}(B)$ by replacing $Y_{i}(u)$ in $\mathbb{Y}_{\varepsilon}(B)$ with $y_{i}(u)$.

Proof. This follows from the exchange relation of a coefficient tuple $y$ by the mutation $\mu_{k}[19]$ :

$$
y_{i}^{\prime}= \begin{cases}y_{k}{ }^{-1}, & i=k, \\ y_{i}\left(1+y_{k}{ }^{-1}\right)^{-B_{k i},}, & i \neq k, \quad B_{k i} \geq 0, \\ y_{i}\left(1+y_{k}\right)^{-B_{k i},} & i \neq k, \quad B_{k i} \leq 0 .\end{cases}
$$

Theorem 6.19. The group $\tilde{y}_{+}^{\circ}(B)_{ \pm}$is isomorphic to $\mathcal{G}_{Y}(B, y)$ by the correspondence $Y_{i}(u) \mapsto$ $y_{i}(u)^{ \pm 1}, 1+Y_{i}(u) \mapsto 1+y_{i}(u)^{ \pm 1}$ for $(i, u)$ satisfying $\mathbf{P}_{ \pm}$. Similarly, the group $\tilde{y}_{-}^{\circ}(B)_{ \pm}$is isomorphic to $\mathcal{G}_{Y}(B, y)$ by the correspondence $Y_{i}(u) \mapsto y_{i}(u)^{\mp 1}, 1+Y_{i}(u) \mapsto 1+y_{i}(u)^{\mp 1}$ for $(i, u)$ satisfying $\mathbf{P}_{ \pm}$. 
Proof. Let us show that $\tilde{y}_{+}^{\circ}(B)_{+} \simeq \mathcal{G}_{Y}(B, y)$. Let $f: \mathbb{Q}_{s f}(y) \rightarrow \tilde{y}_{+}(B)$ be the semifield homomorphism defined by

$$
f: y_{i} \mapsto \begin{cases}Y_{i}(0), & i \in I_{+}, \\ Y_{i}(-1)^{-1}, & i \in I_{-} .\end{cases}
$$

Then, due to Lemma 6.18 (2), it can be shown by induction on $\pm u$ that we have $f: y_{i}(u) \mapsto Y_{i}(u)$ for any $(i, u)$ satisfying $\mathbf{P}_{+}$, and $f: y_{i}(u) \mapsto Y_{i}(u-1)^{-1}$ for any $(i, u)$ satisfying $\mathbf{P}_{-}$. By the restriction of $f$, we have a multiplicative group homomorphism $f^{\prime}: \mathcal{G}_{Y}(B, y) \rightarrow \tilde{y}_{+}^{\circ}(B)_{+}$. On the other hand, by Lemma 6.18 (2) again, a semifield homomorphism $g: \tilde{y}_{+}(B) \rightarrow \mathbb{Q}_{\mathrm{sf}}(y)$ is defined by $Y_{i}(u) \mapsto y_{i}(u)^{ \pm 1}$ for $(i, u)$ satisfying $\mathbf{P}_{ \pm}$. By the restriction of $g$, we have a multiplicative group homomorphism $g^{\prime}: \tilde{y}_{+}^{\circ}(B)_{+} \rightarrow \mathcal{G}_{Y}(B, y)$. Then, $f^{\prime}$ and $g^{\prime}$ are the inverse to each other by Lemma $6.18(1)$. Therefore, $\tilde{y}_{+}^{\circ}(B)_{+} \simeq \mathcal{G}_{Y}(B, y)$. The other cases are similar.

\subsection{Restricted $\mathrm{T}$ and $\mathrm{Y}$-systems and cluster algebras: simply laced case}

The restricted $\mathrm{T}$ and Y-systems, $T_{\ell}(C)$ and $Y_{\ell}(C)$, introduced in Section 5 are special cases of $T(B)$ and $Y_{ \pm}(B)$, if $C$ is simply laced. Therefore, they are also related to cluster algebras.

\subsubsection{Bipartite case}

Suppose that $C$ is a simply laced and bipartite generalized Cartan matrix. Then, we have already seen in Examples 6.8 and 6.9 that $T_{\ell}(C)$ and $Y_{\ell}(C)$ coincides with $T(B)$ and $Y_{\varepsilon}(B)$ for some $B$ and $\varepsilon$. Therefore, we immediately obtain the following results as special cases of Theorems 6.15 and 6.19.

Corollary 6.20. Let $C$ be a simply laced and bipartite generalized Cartan matrix with $I=$ $I_{+} \sqcup I_{-}$. For $\varepsilon \in\{+,-\}$, let $\mathcal{T}_{\ell}^{\circ}(C)_{\varepsilon}$ be the subring of $\mathcal{T}_{\ell}^{\circ}(C)$ for $U=\mathbb{Z}$ generated by $T_{m}^{(a)}(u)$ $(a \in I ; m=1, \ldots, \ell-1 ; u \in \mathbb{Z})$ satisfying $\varepsilon(a)(-1)^{m+1+u}=\varepsilon$. Then, we have the following:

(1) $\mathcal{T}_{2}^{\circ}(C)_{\varepsilon}$ is isomorphic to $\mathcal{A}_{T}(B, x)$ with $B=B(C)$ by the correspondence $T_{1}^{(a)}(u) \mapsto x_{a}(u)$.

(2) For $\ell \geq 3, \mathcal{T}_{\ell}^{\circ}(C)_{\varepsilon}$ is isomorphic to $\mathcal{A}_{T}(B, x)$ with $B=B(C) \square B\left(C^{\prime}\right)$ by the correspondence $T_{m}^{(a)}(u) \mapsto x_{a m}(u)$, where $C^{\prime}$ is the Cartan matrix of type $A_{\ell-1}$ with $I_{+}^{\prime}=\{1,3, \ldots\}$ and $I_{-}^{\prime}=\{2,4, \ldots\}$.

Corollary 6.21. Let $C$ be a simply laced and bipartite generalized Cartan matrix with $I=$ $I_{+} \sqcup I_{-}$. Let $\tilde{y}_{\ell}(C)$ be the semifield with generators $Y_{m}^{(a)}(u)(a \in I ; m=1, \ldots, \ell-1 ; u \in \mathbb{Z})$ and the relations $\mathbb{Y}_{\ell}(C)$ for $U=\mathbb{Z}$. Let $\tilde{y}_{\ell}^{\circ}(C)$ be the multiplicative subgroup of $\tilde{y}_{\ell}(C)$ generated by $Y_{m}^{(a)}(u)$ and $1+Y_{m}^{(a)}(u)(a \in I ; m=1, \ldots, \ell-1 ; u \in \mathbb{Z})$. For $\varepsilon \in\{+,-\}$, let $y_{\ell}^{\circ}(C)_{\varepsilon}$ be the multiplicative subgroup of $\tilde{y}_{\ell}^{\circ}(C)$ generated by $Y_{m}^{(a)}(u)$ and $1+Y_{m}^{(a)}(u)(a \in I ; m=1, \ldots, \ell-1$; $u \in \mathbb{Z})$ satisfying $\varepsilon(a)(-1)^{m+1+u}=\varepsilon$. Then, we have the following:

(1) $\tilde{y}_{2}^{\circ}(C)_{ \pm}$is isomorphic to $\mathcal{G}_{Y}(B, y)$ with $B=B(C)$ by the correspondence $Y_{1}^{(a)}(u) \mapsto$ $y_{a}(u)^{\mp 1}, 1+Y_{1}^{(a)}(u) \mapsto 1+y_{a}(u)^{\mp 1}$.

(2) For $\ell \geq 3$, $\tilde{y}_{\ell}^{\circ}(C)_{ \pm}$is isomorphic to $\mathcal{G}_{Y}(B, y)$ with $B=B(C) \square B\left(C^{\prime}\right)$ by the correspondence $Y_{m}^{(a)}(u) \mapsto y_{a m}(u)^{ \pm 1}, 1+Y_{m}^{(a)}(u) \mapsto 1+y_{a m}(u)^{ \pm 1}$, where $C^{\prime}$ is the Cartan matrix of type $A_{\ell-1}$ with $I_{+}^{\prime}=\{1,3, \ldots\}$ and $I_{-}^{\prime}=\{2,4, \ldots\}$.

The slight discrepancy of the signs between $\ell=2$ and $\ell \geq 3$ is due to the convention adopted here and not an essential problem. 


\subsubsection{Nonbipartite case}

Let us extend Corollaries 6.20 and 6.21 to a simply laced and nonbipartite generalized Cartan matrix $C$. The Cartan matrix of type $A_{2 r}^{(1)}$ is such an example. In general, a generalized Cartan matrix $C$ is bipartite if and only if there is no odd cycle in the corresponding Dynkin diagram. Without loss of generality we can assume that $C$ is indecomposable; namely, the corresponding Dynkin diagram is connected.

Definition 6.22. Let $C=\left(C_{i j}\right)_{i, j \in I}$ be a simply laced, nonbipartite, and indecomposable generalized Cartan matrix. We introduce an index set $I^{\#}=I_{+}^{\#} \sqcup I_{-}^{\#}$, where $I_{+}^{\#}=\left\{i_{+}\right\}_{i \in I}$ and $I_{-}^{\#}=\left\{i_{-}\right\}_{i \in I}$, and define a matrix $C^{\#}=\left(C_{\alpha \beta}^{\#}\right)_{\alpha, \beta \in I^{\#}}$ by

$$
C_{\alpha \beta}^{\#}= \begin{cases}2, & \alpha=\beta, \\ C_{i j}, & (\alpha, \beta)=\left(i_{+}, j_{-}\right) \text {or }\left(i_{-}, j_{+}\right), \\ 0, & \text { otherwise. }\end{cases}
$$

We call $C^{\#}$ the bipartite double of $C$.

It is clear that $C^{\#}$ is a simply laced and indecomposable generalized Cartan matrix; furthermore, it is bipartite with $I^{\#}=I_{+}^{\#} \sqcup I_{-}^{\#}$.

Example 6.23. Let $C$ be the Cartan matrix corresponding to the Dynkin diagram in the left hand side below. Then, $C^{\#}$ is the Cartan matrix corresponding to the Dynkin diagram in the right hand side.
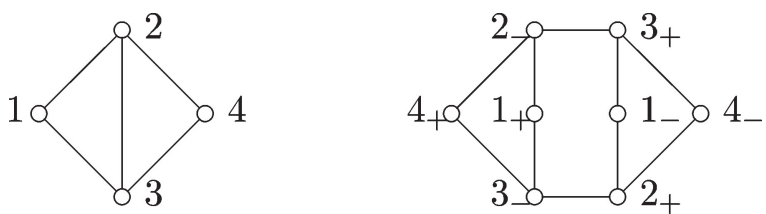

Here is another example.
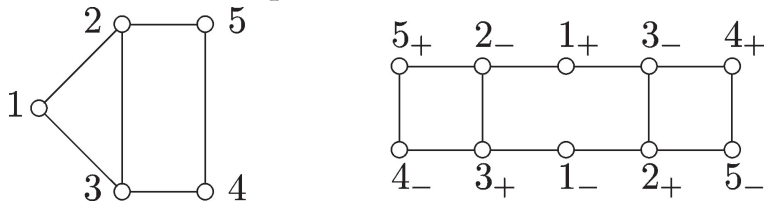

Proposition 6.24. Let $C=\left(C_{i j}\right)_{i, j \in I}$ be a simply laced, nonbipartite, and indecomposable generalized Cartan matrix, and $C^{\#}$ be its bipartite double.

(1) Let $\mathfrak{T}_{\ell}^{\circ}\left(C^{\#}\right)_{+}$be the ring defined in Corollary 6.20. Then, $\mathcal{T}_{\ell}^{\circ}(C)$ is isomorphic to $\mathcal{T}_{\ell}^{\circ}\left(C^{\#}\right)_{+}$ by the correspondence $T_{m}^{(a)}(u) \mapsto T_{m}^{\left(a_{ \pm}\right)}(u)$ for $(-1)^{m+1+u}= \pm$.

(2) Let $\tilde{y}_{\ell}^{\circ}\left(C^{\#}\right)_{+}$be the multiplicative group defined in Corollary 6.21. Then, $\tilde{y}_{\ell}^{\circ}(C)$ is isomorphic to $\tilde{y}_{\ell}^{\circ}\left(C^{\#}\right)_{+}$by the correspondence $Y_{m}^{(a)}(u) \mapsto Y_{m}^{\left(a_{ \pm}\right)}(u), 1+Y_{m}^{(a)}(u) \mapsto 1+Y_{m}^{\left(a_{ \pm}\right)}(u)$ for $(-1)^{m+1+u}= \pm$.

Proof. The generators and relations of the both sides coincide under the correspondence.

Combining Corollaries 6.20, 6.21, and Proposition 6.24, we have the versions of Corollaries 6.20 and 6.21 in the nonbipartite case.

Corollary 6.25. Let $C$ and $C^{\#}$ be the same ones as in Proposition 6.24.

(1) $\mathcal{T}_{2}^{\circ}(C)$ is isomorphic to $\mathcal{A}_{T}(B, x)$ with $B=B\left(C^{\#}\right)$ by the correspondence $T_{1}^{(a)}(u) \mapsto$ $x_{a_{ \pm}}(u)$ for $(-1)^{u}= \pm$.

(2) For $\ell \geq 3, \mathcal{T}_{\ell}^{\circ}(C)$ is isomorphic to $\mathcal{A}_{T}(B, x)$ with $B=B\left(C^{\#}\right) \square B\left(C^{\prime}\right)$ by the correspondence $T_{m}^{(a)}(u) \mapsto x_{a_{ \pm}, m}(u)$ for $(-1)^{m+1+u}= \pm$, where $C^{\prime}$ is the Cartan matrix of type $A_{\ell-1}$ with $I_{+}^{\prime}=\{1,3, \ldots\}$ and $I_{-}^{\prime}=\{2,4, \ldots\}$. 
Corollary 6.26. Let $C$ and $C^{\#}$ be the same ones as in Proposition 6.24.

(1) $\tilde{y}_{2}^{\circ}(C)$ is isomorphic to $\mathcal{G}_{Y}(B, y)$ with $B=B\left(C^{\#}\right)$ by the correspondence $Y_{1}^{(a)}(u) \mapsto$ $y_{a_{ \pm}}(u)^{-1}, 1+Y_{1}^{(a)}(u) \mapsto 1+y_{a_{ \pm}}(u)^{-1}$ for $(-1)^{u}= \pm$.

(2) For $\ell \geq 3$, $\tilde{y}_{\ell}(C)$ is isomorphic to $\mathcal{G}_{Y}(B, y)$ with $B=B\left(C^{\#}\right) \square B\left(C^{\prime}\right)$ by the correspondence $Y_{m}^{(a)}(u) \mapsto y_{a_{ \pm}, m}(u), 1+Y_{m}^{(a)}(u) \mapsto 1+y_{a_{ \pm}, m}(u)$ for $(-1)^{m+1+u}= \pm$, where $C^{\prime}$ is the Cartan matrix of type $A_{\ell-1}$ with $I_{+}^{\prime}=\{1,3, \ldots\}$ and $I_{-}^{\prime}=\{2,4, \ldots\}$.

\subsection{Concluding remarks}

One can further extend Corollaries 6.20,6.21, 6.25, and 6.26 to the tamely laced and nonsimply laced case by introducing $\mathrm{T}$ and $\mathrm{Y}$-systems associated with another class of cluster algebras ${ }^{1}$. Therefore, we conclude that all the restricted $\mathrm{T}$ and $\mathrm{Y}$-systems associated with tamely laced generalized Cartan matrices introduced in Section 5 are identified with the $\mathrm{T}$ and Y-systems associated with a certain class of cluster algebras.

The following question is left as an important problem: What are the $\mathrm{T}$ and $\mathrm{Y}$-systems associated with nontamely laced symmetrizable generalized Cartan matrices?

\section{Acknowledgements}

It is our great pleasure to thank Professor Tetsuji Miwa on the occasion of his sixtieth birthday for his generous support and continuous interest in our works through many years. We also thank David Hernandez for a valuable comment.

\section{References}

[1] Batchelor M.T., Guan X.-W., Oelkers N., Tsuboi Z., Integrable models and quantum spin ladders: comparison between theory and experiment for the strong coupling ladder compounds, Adv. Phys. 56 (2007), 465-543, cond-mat/0512489.

[2] Bazhanov V.V., Lukyanov S.L., Zamolodchikov A.B., Integrable structure of conformal field theory, quantum KdV theory and thermodynamic Bethe ansatz, Comm. Math. Phys. 177 (1996), 381-398, hep-th/9412229.

[3] Bazhanov V.V., Reshetikhin N., Restricted solid-on-solid models connected with simply laced algebras and conformal field theory, J. Phys. A: Math. Gen. 23 (1990), 1477-1492.

[4] Beck J., Braid group action and quantum affine algebras, Comm. Math. Phys. 165 (1994), 555-568, hep-th/9404165.

[5] Berenstein A., Fomin S., Zelevinsky A., Cluster algebras. III. Upper bounds and double Bruhat cells, Duke Math. J. 126 (2005), 1-52, math.RT/0305434.

[6] Caracciolo R., Gliozzi R., Tateo R., A topological invariant of RG flows in 2D integrable quantum field theories, Internat. J. Modern Phys. B 13 (1999), 2927-2932, hep-th/9902094.

[7] Chari V., Pressley A., Quantum affine algebras, Comm. Math. Phys. 142 (1991), 261-283.

[8] Chari V., Pressley A., Quantum affine algebras and their representations, in Representations of Groups (Banff, AB, 1994), CMS Conf. Proc., Vol. 16, Amer. Math. Soc., Providence, RI, 1995, 59-78, hep-th/9411145.

[9] Chari V., Pressley A., Minimal affinizations of representations of quantum groups: the simply laced case, J. Algebra 184 (1996), 1-30, hep-th/9410036.

[10] Di Francesco P., Kedem R., Q-systems as cluster algebras II: Cartan matrix of finite type and the polynomial property, Lett. Math. Phys. 89 (2009), 183-216, arXiv:0803.0362.

[11] Di Francesco P., Kedem R., Positivity of the T-system cluster algebra, arXiv:0908.3122.

[12] Dorey P., Dunning C., Tateo R., The ODE/IM correspondence, J. Phys. A: Math. Theor. 40 (2007), R205R283, hep-th/0703066.

\footnotetext{
${ }^{1}$ Inoue R., Iyama O., Keller B., Kuniba A., Nakanishi T., In preparation.
} 
[13] Dorey P., Pocklington A., Tateo R., Integrable aspects of the scaling $q$-state Potts models. II. Finite-size effects, Nuclear Phys. B 661 (2003), 464-513, hep-th/0208202.

[14] Drinfel'd V., Hopf algebras and the quantum Yang-Baxter equation, Soviet. Math. Dokl. 32 (1985), $254-258$.

[15] Drinfel'd V., A new realization of Yangians and quantized affine algebras, Soviet. Math. Dokl. 36 (1988), $212-216$.

[16] Fomin S., Zelevinsky A., Cluster algebras. I. Foundations, J. Amer. Math. Soc. 15 (2002), 497-529, math.RT/0104151.

[17] Fomin S., Zelevinsky A., Cluster algebras. II. Finite type classification, Invent. Math. 154 (2003), 63-121, math.RA/0208229.

[18] Fomin S., Zelevinsky A., Y-systems and generalized associahedra, Ann. of Math. (2) 158 (2003), 977-1018, hep-th/0111053.

[19] Fomin S., Zelevinsky A., Cluster algebras. IV. Coefficients, Compos. Math. 143 (2007), 112-164, math.RA/0602259.

[20] Frenkel E., Reshetikhin N., The $q$-characters of representations of quantum affine algebras and deformations of $W$-algebras, in Recent Developments in Quantum Affine Algebras and Related Topics (Raleigh, NC, 1998), Contemp. Math., Vol. 248, Amer. Math. Soc., Providence, RI, 1999, 163-205, math.QA/9810055.

[21] Frenkel E., Szenes A., Thermodynamic Bethe ansatz and dilogarithm identities. I, Math. Res. Lett. 2 (1995), 677-693, hep-th/9506215.

[22] Gliozzi F., Tateo R., Thermodynamic Bethe ansatz and three-fold triangulations, Internat. J. Modern Phys. A 11 (1996), 4051-4064, hep-th/9505102.

[23] Geiss C., Leclerc B., Schröer J., Cluster algebra structures and semicanonical bases for unipotent groups, math.RT/0703039.

[24] Gromov N., Kazakov V., Vieira P., Finite volume spectrum of 2D field theories from Hirota dynamics, arXiv:0812.5091.

[25] Hernandez D., The Kirillov-Reshetikhin conjecture and solutions of T-systems, J. Reine Angew. Math. 596 (2006), 63-87, math.QA/0501202.

[26] Hernandez D., Representations of quantum affinizations and fusion product, Transform. Groups 10 (2005), 163-200, math.QA/0312336.

[27] Hernandez D., Drinfeld coproduct, quantum fusion tensor category and applications, Proc. Lond. Math. Soc. (3) 95 (2007), 567-608, math.QA/0504269.

[28] Hernandez D., The Kirillov-Reshetikhin conjecture: the general case, arXiv:0704.2838.

[29] Hernandez D., Leclerc B., Cluster algebras and quantum affine algebras, talk presented by B. Leclerc at Workshop "Lie Theory" (MSRI, Berkeley, March 2008).

[30] Hernandez D., Leclerc B., Cluster algebras and quantum affine algebras, arXiv:0903.1452.

[31] Hutchins H.C., Weinert H.J., Homomorphisms and kernels of semifields, Period. Math. Hungar. 21 (1990), 113-152.

[32] Inoue R., Iyama O., Kuniba A., Nakanishi T., Suzuki J., Periodicities of T-systems and Y-systems, Nagoya Math. J., to appear, arXiv:0812.0667.

[33] Jimbo M., A q-difference analogue of $U(\hat{\mathfrak{g}})$ and the Yang-Baxter equation, Lett. Math. Phys. 10 (1985), 63-69.

[34] Jing N., Quantum Kac-Moody algebras and vertex representations, Lett. Math. Phys. 44 (1998), $261-271$.

[35] Kac V.G., Infinite-dimensional Lie algebras, 3rd ed., Cambridge University Press, Cambridge, 1990.

[36] Keller B., Cluster algebras, quiver representations and triangulated categories, arXiv:0807.1960.

[37] Kirillov A.N., Identities for the Rogers dilogarithm function connected with simple Lie algebras, J. Soviet Math. 47 (1989), 2450-2459.

[38] Kirillov A.N., Reshetikhin N., Representations of Yangians and multiplicities of the inclusion of the irreducible components of the tensor product of representations of simple Lie algebras, J. Soviet Math. 52 (1990), 3156-3164.

[39] Klassen T.R., Melzer E., Purely elastic scattering theories and their ultraviolet limits, Nuclear Phys. B 338 (1990), 485-528. 
[40] Klümper A., Pearce P.A., Conformal weights of RSOS lattice models and their fusion hierarchies, Phys. A 183 (1992), 304-350.

[41] Krichever I., Lipan O., Wiegmann P., Zabrodin A., Quantum integrable models and discrete classical Hirota equations. Comm. Math. Phys. 188 (1997), 267-304.

[42] Kuniba A., Nakanishi T., Spectra in conformal field theories from the Rogers dilogarithm, Internat. J. Modern Phys. A 7 (1992), 3487-3494, hep-th/9206034.

[43] Kuniba A., Nakanishi T., Suzuki J., Functional relations in solvable lattice models. I. Functional relations and representation theory, Internat. J. Modern Phys. A 9 (1994), 5215-5266, hep-th/9309137.

[44] Kuniba A., Nakanishi T., Suzuki J., Functional relations in solvable lattice models. II. Applications, Internat. J. Modern Phys. A 9 (1994), 5267-5312, hep-th/9310060.

[45] Kuniba A., Suzuki J., Functional relations and analytic Bethe ansatz for twisted quantum affine algebras, J. Phys. A: Math. Gen. 28 (1995), 711-722, hep-th/9408135.

[46] Miki K., Representations of quantum toroidal algebra $U_{q}\left(s l_{n+1, \text { tor }}\right)(n \geq 2)$, J. Math. Phys. 41 (2000), 7079-7098.

[47] Nakajima H., Quiver varieties and finite dimensional representations of quantum affine algebras, J. Amer. Math. Soc. 14 (2001), 145-238, math.QA/9912158.

[48] Nakajima H., $t$-analogs of $q$-characters of Kirillov-Reshetikhin modules of quantum affine algebras, Represent. Theory 7 (2003), 259-274, math.QA/0009231.

[49] Nakajima H., Quiver varieties and cluster algebras, arXiv:0905.0002.

[50] Noumi M., Yamada Y., Affine Weyl groups, discrete dynamical systems and Painlevé equations, Comm. Math. Phys. 199 (1998), 281-295, math.QA/9804132.

[51] Noumi M., Yamada Y., Birational Weyl group action arising from a nilpotent Poisson algebra, in Proc. of the Nagoya 1999 International Workshop "Physics and Combinatorics 1999" (Nagoya), World Sci. Publ., River Edge, NJ, 2001, 287-319.

[52] Ravanini R., Valleriani A., Tateo R., Dynkin TBA's, Internat. J. Modern Phys. A 8 (1993), 1707-1727, hep-th/9207040.

[53] Runkel I., Perturbed defects and T-systems in conformal field theory, J. Phys. A: Math. Theor. 41 (2008), 105401, 21 pages, arXiv:0711.0102.

[54] Tsuboi Z., Solutions of discretized affine Toda field equations for $A_{n}^{(1)}, B_{n}^{(1)}, C_{n}^{(1)}, D_{n}^{(1)}, A_{n}^{(2)}$ and $D_{n+1}^{(2)}$, J. Phys. Soc. Japan 66 (1997), 3391-3398, solv-int/9610011.

[55] Tsuboi Z., Solutions of the T-system and Baxter equations for supersymmetric spin chains, Nuclear Phys. B 826 (2010), 399-455, arXiv:0906.2039.

[56] Varagnolo M., Vasserot E., Schur duality in the toroidal setting, Comm. Math. Phys. 182 (1996), 469-483, q-alg/9506026.

[57] Volkov A.Yu., On the periodicity conjecture for Y-systems, Comm. Math. Phys. 276 (2007), 509-517, hep-th/0606094.

[58] Zamolodchikov Al.B., On the thermodynamic Bethe ansatz equations for reflectionless ADE scattering theories, Phys. Lett. B 253 (1991), 391-394. 